\title{
Neutrino-nucleus scattering in the SuSA model
}

\author{
J. E. Amaro ${ }^{1}$, M. B. Barbaro ${ }^{2,3, a}$ (I) , J. A. Caballero ${ }^{4,5}$, T. W. Donnelly ${ }^{6}$, R. González-Jiménez ${ }^{7}$, G. D. Megias ${ }^{4,8}$, \\ and I. Ruiz Simo ${ }^{1}$ \\ ${ }^{1}$ Departamento de Física Atómica, Molecular y Nuclear, and Instituto de Física Teórica y Computacional Carlos I, \\ Universidad de Granada, 18071 Granada, Spain \\ 2 Dipartimento di Fisica, Università di Torino and INFN, Sezione di Torino, Via P. Giuria 1, 10125 Turin, Italy \\ 3 Université Paris-Saclay, CNRS/IN2P3, IJCLab, 91405 Orsay, France \\ 4 Departamento de Física Atómica, Molecular y Nuclear, Universidad de Sevilla, 41080 Seville, Spain \\ 5 Instituto de Física Teórica y Computacional Carlos I, 18071 Granada, Spain \\ ${ }^{6}$ Laboratory for Nuclear Science, Department of Physics, Center for Theoretical Physics, Massachusetts Institute of \\ Technology, Cambridge, MA 02139, USA \\ 7 Grupo de Física Nuclear, Departamento de Estructura de la Materia, Física Térmica y Electrónica, Facultad de Ciencias \\ Físicas, Universidad Complutense de Madrid and IPARCOS, 28040 Madrid, Spain \\ 8 Research Center for Cosmic Neutrinos, Institute for Cosmic Ray Research, University of Tokyo, Kashiwa, Japan
}

Received 5 June 2021 / Accepted 19 September 2021 / Published online 25 October 2021 (C) The Author(s) 2021

\begin{abstract}
The super-scaling approach (SuSA) model, based on the analogies between electron and neutrino interactions with nuclei, is reviewed and its application to the description of neutrino-nucleus scattering is presented. The contribution of both one- and two-body relativistic currents is considered. The model is validated with the $\left(e, e^{\prime}\right)$ data, including also inelastic contributions for the inclusive reaction. A discussion of semi-inclusive reactions and their implications for charge-changing reactions of neutrinos is also presented. A selection of results for the inclusive neutrino reactions with change of charge is presented where theoretical predictions are compared with cross-section measurements from the main ongoing neutrino oscillation experiments.
\end{abstract}

\section{Introduction}

A huge experimental programme on accelerator-based neutrino oscillation experiments has been developed in recent years with the scope of improving our knowledge of neutrino properties and of potentially answering one of the fundamental open questions of modern physics, that is the origin of the matter/antimatter asymmetry in the universe, by measuring the weak CPviolating phase [1]. The success of this program partly relies on the control of systematic errors, largely due to uncertainties in the description of the neutrino interactions with the detector, typically made of mediumweight nuclei like carbon, oxygen or argon. With this motivation, intense theoretical activity has been carried out in parallel to provide an accurate description of neutrino-nucleus reactions in the $\mathrm{GeV}$ region, relevant for the experiments, for different processes ranging from quasielastic up to deep inelastic scattering, encompassing the excitation of nucleon resonances and the emission of two or more nucleons (see Ref. [2] for a comprehensive review of recent progresses and open challenges in the field).

\footnotetext{
${ }^{a}$ e-mail: barbaro@to.infn.it (corresponding author)
}

The scattering of energetic neutrinos with atomic nuclei has been studied in depth from a theoretical point of view for more than a decade. There is abundant bibliography and review articles have been written on the subject [3-10]. However, the problem is non-trivial and there are still differences between the different existing theoretical models. The reasons are many and profound. The main reason is that the atomic nucleus is a system of many strongly interacting particles that undergo the Pauli exclusion principle. This implies that the probability of interaction with a nucleus is not the sum of the probabilities of interaction with its components (nucleons). The nucleons are not free particles but they are correlated with each other and the interaction in the final state of the hadrons that emerge from the reaction (nucleons, pions, ...) must also be accounted for.

Furthermore, the energies of the neutrinos in the accelerators are of the order of the $\mathrm{GeV}$ and this implies large transfers of energy and momentum to the nucleus, which requires a relativistic treatment of the nuclear problem. In this regard, the models that treat relativity in an appropriate way have been the most used (relativistic fermi gas, relativistic mean field).

When it comes to the interaction of neutrinos with many-particle systems, there is the added difficulty of 
multinucleon emission, either due to the interaction with two correlated nucleons, or due to the action of meson exchange currents (MEC). The main emission channel of two nucleons $(2 \mathrm{p} 2 \mathrm{~h})$ can represent more than $20 \%$ of the quasi-elastic cross section depending on the kinematics. Its main contribution comes from the excitation of a $\Delta$ that within the medium can decay, emitting two nucleons. The excitation of the $\Delta$ is also the largest contribution to the pion emission channel, where the interaction in the final state of the pions must also be taken into account.

An alternative to microscopic nuclear models are the phenomenological scaling models applied to the quasielastic response functions. The phenomenon of scaling is sometimes found to occur in different fields, including solid state, atomic, molecular, nuclear and hadronic physics, when an interacting probe scatters from composite many-body systems. Under some circumstances, it is found that the response of the complex system no longer depends on two independent variables, but only on a particular combination of those, called the scaling variable. This phenomenon is very well known in high energy physics where the inelastic nucleon response functions are shown to depend only on the Bjorken variable $x$ [11]. A similar phenomenon is observed in lepton-nucleus scattering, where the nuclear response functions are found to scale with a single scaling variable, denoted as $y$. This indicates that the probe (lepton) interacts with the nucleus' constituents, in this case with the nucleons in the nucleus.

The phenomenon of $y$-scaling emerges from the analysis of quasielastic (QE) $\left(e, e^{\prime}\right)$ reactions and has been studied in detail in Refs. [12-14]. The scaling function is defined as the $\mathrm{QE}\left(e, e^{\prime}\right)$ differential cross section divided by a single-nucleon cross section averaged over the Fermi gas. For high enough values of the momentum transfer, $q$, the scaling function does only depend on a single variable, $y$, given as a particular combination of the energy $(\omega)$ and momentum $(q)$ transferred in the process. The scaling variable $y$ is (up to a sign) the minimum value of the missing momentum allowed by kinematics [12-14].

In this article, we review our work on the SuSA (super-scaling approach) model, originally developed in Ref. [15] and subsequently refined and improved to the updated version SuSAv2 [16,17] (see also Refs. [18,19] for other recent reviews). The original SuSA, as will be explained in more detail in Sect. 2.2, is a phenomenological model which, while retaining the relativistic aspect of the relativistic fermi gas (RFG), provides by construction - unlike the RFG - a good description of inclusive electron scattering data $\left(e, e^{\prime}\right)$ in the quasielastic region. In the model initial and final state interaction effects, absent in the RFG, are directly extracted from $\left(e, e^{\prime}\right)$ data. The updated version, SuSAv2, implements inputs from the relativistic mean field model (RMF), which provides a microscopic interpretation of the basic features of SuSA and also includes a more detailed description of the different spin and isospin channels.
Moreover, the model has been extended to include two-body currents, able to excite two-particle-two-hole $(2 \mathrm{p} 2 \mathrm{~h})$ states; these, as will be shown in Sect. 4, play an important role in the analysis of neutrino-nucleus experimental data.

The scheme of this review is the following. In Sect. 2 we focus on the inclusive reaction, presenting the formalism, describing the model and testing it with electron scattering data. In Sect. 3 we discuss the semiinclusive reaction an its relation to the inclusive one. In Sect. 4 we show some selected results and discuss the comparison with experimental data. Finally, in Sect. 5 we draw our conclusion and outline the future developments of our work.

\section{Inclusive neutrino-nucleus scattering}

\subsection{General formalism}

In this review, we mainly focus on the charged current $(\mathrm{CC})$ inclusive process

$$
\nu_{l}\left(\bar{\nu}_{l}\right)+A \longrightarrow l^{-}\left(l^{+}\right)+X,
$$

where a neutrino (antineutrino) of a given flavour $l$ having four-momentum $K^{\mu}=(\varepsilon, \mathbf{k})$ hits a nucleus $A$ and a charged lepton $l^{-}\left(l^{+}\right)$is detected in the final state with four-momentum $K^{\prime \mu}=\left(\varepsilon^{\prime}, \mathbf{k}^{\prime}\right)$ and scattering angle $\theta$, while the residual system $X$ is unobserved. The four-momentum transferred from the probe to the nucleus is $Q^{\mu}=(\omega, \mathbf{q})$, with $\omega=\varepsilon-\varepsilon^{\prime}$ and $\mathbf{q}=\mathbf{k}-\mathbf{k}^{\prime}$. We shall assume the neutrino to be massless, while the outgoing lepton has finite mass $m_{l}$. The corresponding cross section is obtained by contracting the leptonic and hadronic tensors

$$
\begin{aligned}
& l_{\mu \nu}(\mathbf{q}, \omega) \\
& \quad=2\left(K_{\mu} K_{\nu}^{\prime}+K_{\mu}^{\prime} K_{\nu}-K \cdot K^{\prime} g_{\mu \nu}+i \chi \epsilon_{\mu \nu \rho \sigma} K^{\rho} K^{\prime \sigma}\right) \\
& W^{\mu \nu}(\mathbf{q}, \omega) \\
& \quad=\sum_{n}<A\left|J^{\mu \dagger}(\mathbf{q}, \omega)\right| n><n\left|J^{\nu}(\mathbf{q}, \omega)\right| A> \\
& \quad \delta\left(\omega+E_{A}-E_{n}\right),
\end{aligned}
$$

where $\chi=+1(-1)$ in the neutrino (antineutrino) case, $\mid A>$ is the initial nuclear ground state and $|n\rangle$ are all the intermediate hadronic states that can be reached through the weak current operator $J^{\mu}$. The double differential cross section with respect to the momentum and scattering angle of the outgoing lepton can be expressed in terms of five response functions [15] (for the semi-inclusive reaction $\left(\nu_{l}, l^{-} N\right)$, which will be briefly discussed in Sect. 3, the response functions are ten) 


$$
\begin{aligned}
\frac{d^{2} \sigma}{d \varepsilon^{\prime} d \cos \theta}= & \frac{\left(G_{F} \cos \theta_{c}\right)^{2}}{4 \pi} v_{0} \frac{k^{\prime}}{\varepsilon^{\prime}}\left(V_{C C} R_{C C}+2 V_{C L} R_{C L}\right. \\
& \left.+V_{L L} R_{L L}+V_{T} R_{T}+2 \chi V_{T^{\prime}} R_{T^{\prime}}\right)
\end{aligned}
$$

where $G_{F}$ the Fermi weak constant, $\theta_{c}$ the Cabibbo angle and $v_{0}=\left(\varepsilon+\varepsilon^{\prime}\right)^{2}-\mathbf{q}^{2}$. The indices $C, L, T$ refer to the Coulomb, longitudinal and transverse components of the leptonic and hadronic currents with respect to $\mathbf{q}$.

The leptonic coefficients $V_{K}$ are related to the components of the tensor (2) and are given by

$$
\begin{aligned}
V_{C C} & =1-\delta^{2} \frac{\left|Q^{2}\right|}{v_{0}}, V_{C L}=\frac{\omega}{q}+\frac{\delta^{2}}{\rho^{\prime}} \frac{\left|Q^{2}\right|}{v_{0}}, \\
V_{L L} & =\frac{\omega^{2}}{q^{2}}+\delta^{2} \frac{\left|Q^{2}\right|}{v_{0}}\left(1+\frac{2 \omega}{q \rho^{\prime}}+\rho \delta^{2}\right), \\
V_{T} & =\frac{\left|Q^{2}\right|}{v_{0}}+\frac{\rho}{2}-\delta^{2} \frac{\left|Q^{2}\right|}{v_{0}}\left(\frac{\omega}{q \rho^{\prime}}+\frac{\rho \delta^{2}}{2}\right), \\
V_{T^{\prime}} & =\frac{\left|Q^{2}\right|}{v_{0}}\left(\frac{1}{\rho^{\prime}}-\frac{\omega \delta^{2}}{q}\right),
\end{aligned}
$$

where the variables $\delta^{2}=\frac{m_{l}^{2}}{\left|Q^{2}\right|}, \rho=\frac{\left|Q^{2}\right|}{q^{2}}$ and $\rho^{\prime}=\frac{q}{\varepsilon+\varepsilon^{\prime}}$ have been introduced.

The response functions

$$
\begin{aligned}
R_{C C} & =W^{00}, R_{C L}=-\frac{1}{2}\left(W^{03}+W^{30}\right), \\
R_{L L} & =W^{33}, R_{T}=W^{11}+W^{22} \\
R_{T^{\prime}} & =-\frac{i}{2}\left(W^{12}-W^{21}\right)
\end{aligned}
$$

embody the nuclear dynamics and are specific components of the hadronic tensor $W^{\mu \nu}$, depending only upon $\omega$ and $\mathbf{q}$.

The above decomposition into response functions is valid for all reaction channels - elastic, quasielastic, inelastic - each of which characterized by a different current operator $J^{\mu}$ in the hadronic tensor (3). In the case of quasielastic scattering, corresponding to the interaction of the probe with a single nucleon, the weak current operator is

$$
\begin{aligned}
J^{\mu}(\mathbf{q}, \omega)= & F_{1}\left(Q^{2}\right) \gamma^{\mu}+\frac{i F_{2}\left(Q^{2}\right)}{2 m_{N}} \sigma^{\mu \nu} Q_{\nu}-G_{A}\left(Q^{2}\right) \gamma^{\mu} \gamma_{5} \\
& -\frac{G_{P}\left(Q^{2}\right)}{m_{N}} Q^{\mu} \gamma_{5}
\end{aligned}
$$

where $F_{1}, F_{2}, G_{A}$ and $G_{P}$ are the Pauli, Dirac, axial and pseudoscalar weak form factors, respectively.

In neutrino oscillation experiments the incident beam is not monochromatic. As a consequence, before comparing with experimental data, the cross section (4) must be folded with the normalized neutrino flux $\phi(\varepsilon)$ $\left(\int d \varepsilon \phi(\varepsilon)=1\right)$ :

$$
\left\langle\frac{d^{2} \sigma}{d \varepsilon^{\prime} d \cos \theta}\right\rangle=\int d \varepsilon \phi(\varepsilon) \frac{d^{2} \sigma}{d \varepsilon^{\prime} d \cos \theta} .
$$

\subsection{Scaling and super-scaling: the SuSAv2 model}

In the $\mathrm{QE}$ domain the basic mechanism in $\left(e, e^{\prime}\right)$ reactions on nuclei corresponds to elastic scattering from individual nucleons in the nuclear medium. This implies that the inclusive $\left(e, e^{\prime}\right)$ cross section is mainly constructed from the exclusive $\left(e, e^{\prime} N\right)$ process, including the contribution of all nucleons in the target and integrating over all (unobserved) ejected nucleon variables (see also the following section). This approach constitutes the basis of the impulse approximation (IA). Thus, the double differential $\left(e, e^{\prime}\right)$ inclusive cross section is given as the sum of two response functions corresponding to the longitudinal, $L$, and transverse, $T$, channels,

$$
\frac{d^{2} \sigma}{d \Omega_{e} d \omega}=\sigma_{\mathrm{Mott}}\left[v_{L} R_{L}(q, \omega)+v_{T} R_{T}(q, \omega)\right]
$$

where $\sigma_{\text {Mott }}$ is the Mott cross section and the $v_{L, T}$ are kinematical factors that involve leptonic variables (see Refs. [18,20] for explicit expressions). In terms of the scaling functions the nuclear responses are

$$
R_{L, T}(q, \omega)=\frac{1}{k_{F}}\left[f_{L, T}(q, \omega) G_{L, T}(q, \omega)\right],
$$

where $k_{F}$ is the Fermi momentum and $G_{L, T}$ are defined as the responses of a moving nucleon and include relativistic corrections arising from the presence of the medium. Their explicit expressions can be found in Refs. $[16,18,21]$. The terms $f_{L, T}$ are the scaling functions that show a mild dependence upon the momentum transfer $q$ (first-kind scaling) and very weak dependence on the nuclear system considered (second-kind scaling). The occurrence of both types of scaling is denoted as superscaling. Hence to the extent that at some kinematics the above $f$ functions are the same for all nuclei and do only depend on a single variable, denoted as the scaling variable $\psi(q, \omega)$, but not separately on $q$, one says that superscaling occurs. The superscaling variable $\psi$ is given in terms of $q$ and $\omega$ as

$$
\psi=\frac{1}{\sqrt{\xi_{F}}} \frac{\lambda-\tau}{\sqrt{(1+\lambda) \tau+\kappa \sqrt{\tau(1+\tau)}}},
$$

where the dimensionless Fermi kinetic energy $\xi_{F}=\frac{T_{F}}{m_{N}}$, energy transfer $\lambda=\frac{\omega}{2 m_{N}}$, momentum transfer $\kappa=\frac{q}{2 m_{N}}$ and squared four-momentum transfer $\tau=\kappa^{2}-\lambda^{2}$ have been introduced. Here the $y$-variable introduced above is given approximately by

$$
y \cong k_{F} \psi,
$$

as discussed in Ref. [14]. A phenomenological energy shift $E_{\text {shift }}$, fitted for each nucleus to the experimental position of the quasielastic peak [22], is included in the definition of the scaling variable $\psi^{\prime} \equiv \psi\left(q, \omega-E_{\text {shift }}\right)$. 


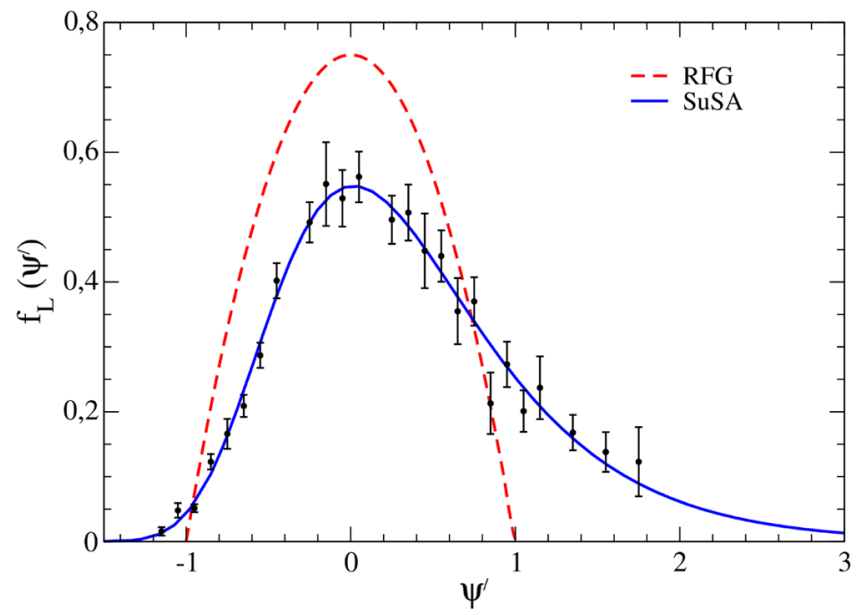

Fig. 1 Left panel: Phenomenological SuSA scaling function versus $\psi^{\prime}$ in the $\mathrm{QE}$ region together with $\left(e, e^{\prime}\right)$ longitudinal scaling data from Ref. [26]. The RFG scaling function is also shown as reference. Right panel: RMF longitudinal

Scaling and superscaling properties of electronnucleus interactions have been analyzed in detail in a series of previous works [12-14,22-25]. The importance of this phenomenon to test the validity of any nuclear model aiming to describe electron scattering reactions has been clearly proven. The model, denoted as the super-scaling approach (SuSA), is entirely based on the phenomenology, making use of a unique, universal, scaling function extracted from the analysis of the longitudinal electron scattering data (see Fig. 1). Notice that the behavior and properties of the experimental superscaling function constitute a strong constraint for any theoretical model describing QE electron scattering. Not only should the superscaling behavior be fulfilled, but also the specific shape of the longitudinal scaling function, $f_{L}^{\exp }$, must be reproduced. The SuSA model assumes the longitudinal phenomenological scaling function to be valid also in describing the transverse channel, i.e., $f_{L}=f_{T}$.

In recent years, we have explored in detail the extension of SuSA to neutrino-nucleus scattering. Due to the complexity of the weak process, with an increased number of nuclear responses compared with the pure electromagnetic one, and the particular role played by the axial term in the weak current, we have developed an improved version of the superscaling model, called SuSAv2 [16]. Contrary to the original SuSA [15,21,27] where a universal scaling function based on electron scattering data is used, the new SuSAv2 model incorporates relativistic mean field (RMF) effects $[24,25]$ in the longitudinal and transverse nuclear responses, as well as in the isovector and isoscalar channels that is of great importance to describe charged-current (CC) neutrino reactions that are purely isovector [28].

The origin of the SuSAv2 approach is based on the capability of the RMF to describe properly the scaling behavior of the electron scattering data. As shown in previous works $[24,25], \mathrm{RMF}$ is one of the few micro-

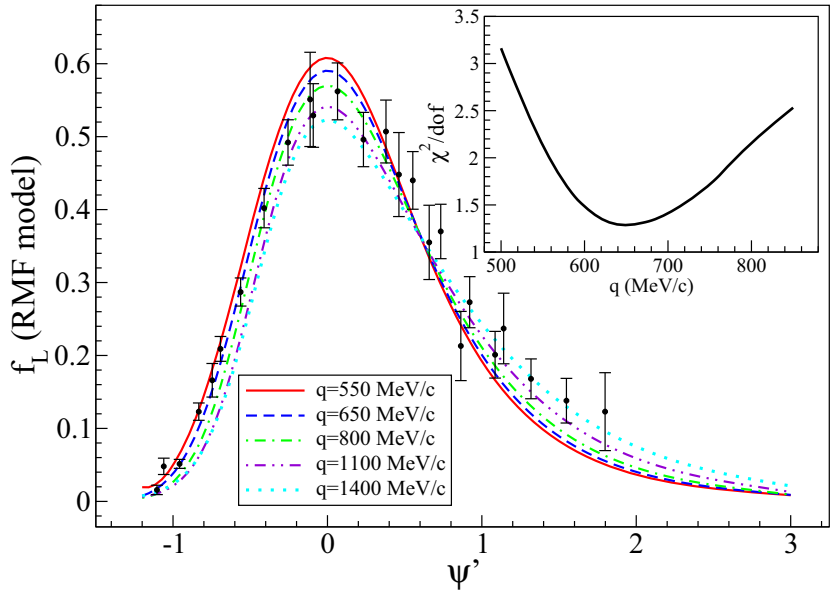

scaling functions for $\left(e, e^{\prime}\right)$ at different $q$ values compared with $\left(e, e^{\prime}\right)$ longitudinal scaling data from Ref. [26]. In the inner smaller plot a reduced- $\chi^{2}$ analyses shows a minimum at $q=650 \mathrm{MeV} / \mathrm{c}$. Figure adapted from Ref. [16]

scopic models capable of reproducing the asymmetric shape of the phenomenological scaling function with a long tail extended to high values of the transfer energy (large values of $\psi^{\prime}$ ). Moreover, RMF produces an enhancement in the transverse scaling function, a genuine dynamical relativistic effect linked to the lower components in the wave functions, that is supported by the analysis of data. The RMF framework to finite nuclei has proven to successfully reproduce the scaling behavior shown by data at low to intermediate $q$ values (see Fig. 1). However, the model clearly fails at higher momentum transfers where final state interactions (FSI) are expected to be weaker. This is due to the RMF strong energy-independent scalar and vector potentials used in the final state that lead to too much asymmetry in the scaling functions and shift the QE peak to very high transfer energies, in clear disagreement with data. To remedy this shortfall of the RMF model, the SuSAv2 incorporates both the RMF scaling functions at low-to-intermediate $q$ values and the relativistic plane wave impulse approximation (RPWIA) ones at higher $q$ using a $q$-dependent blending function that smoothly connects the two regimes (see Refs. $[17,29]$ for details). A similar solution to this drawback of the RMF model has been taken in the recent energydependent RMF (ED-RMF) approach $[30,31]$ where RMF potentials are multiplied by a blending function inspired by the SuSAv2 one that scales them down as the kinetic energy of the scattered nucleon increases, also preventing non-orthogonality issues. This model predicts both lepton and nucleon kinematics, showing a similar agreement on electron and neutrino data with SuSAv2.

In summary, the SuSAv2 model and, for extension, the ED-RMF one reproduce the experimental longitudinal scaling data, gives rise to an enhancement in the electromagnetic transverse channel, i.e., $f_{T}^{\left(e, e^{\prime}\right)}>f_{L}^{\left(e, e^{\prime}\right)}$, takes into account the differences in the 


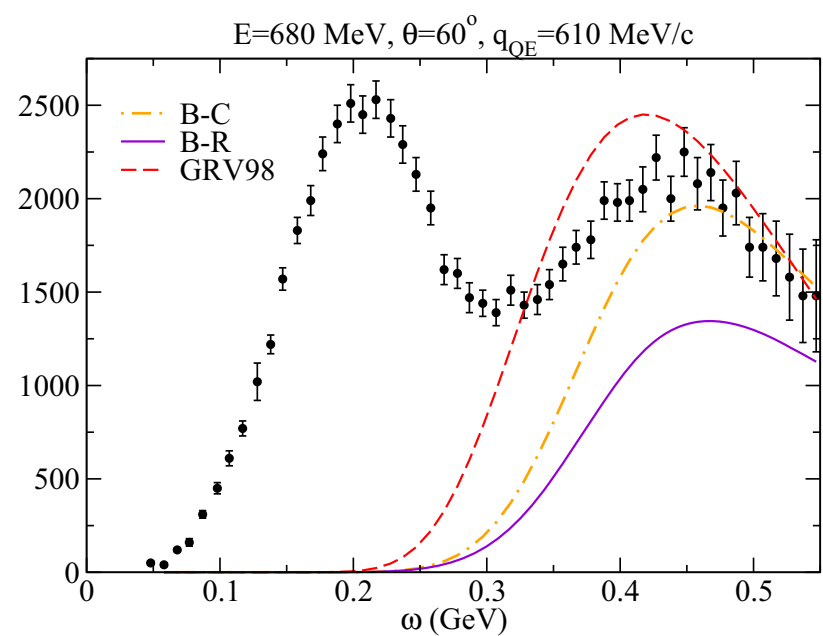

Fig. 2 Comparison of inclusive ${ }^{12} \mathrm{C}\left(e, e^{\prime}\right)$ double differential cross sections and predictions for the inelastic regime of the Bosted-Christy parametrization [33,34] (dot-dashed lines), Bodek-Ritchie parametrization [35,36] (solid lines) and GRV98 PDFs [37,38] (dashed lines) at different kine-

isoscalar/isovector scaling functions, of crucial interest for neutrino scattering processes, and finally avoids the problems of the RMF model in the region of high momentum transfer, where FSI effects are negligible. One of the basic merits of SuSAv2 is the translation of sophisticated and demanding microscopic calculations into relatively straightforward parametrizations and, hence, easing its implementation in the MonteCarlo simulations employed in the analysis of neutrino oscillation experiments.

To conclude, it is important to point out that SuSAv2 is not restricted to the QE kinematics domain. On the contrary, it can be extended to the inelastic region by generalizing the superscaling variable (11) to the excitation of any inelastic state having invariant mass $W$. One can thus define [32]

$$
\begin{aligned}
& \psi_{W}=\frac{1}{\sqrt{\xi_{F}}} \frac{\lambda-\tau \rho_{W}}{\sqrt{\left(1+\lambda \rho_{W}\right) \tau+\kappa \sqrt{\tau\left(1+\tau \rho_{W}^{2}\right)}}}, \\
& \rho_{W}=1+\frac{1}{4 \tau}\left(W^{2} / m_{N}^{2}-1\right)
\end{aligned}
$$

and evaluate the inelastic hadronic tensor as the integral

$$
W_{\text {inel }}^{\mu \nu}=\frac{1}{m_{N}^{2}} \int_{W_{\min }}^{W_{\max }} d W W f\left(\psi_{W}\right) w_{\text {inel }}^{\mu \nu}
$$

between the limits $W_{\min }, W_{\max }$ imposed by the kinematics, where $f\left(\psi_{W}\right)$ is the SuSAv2 superscaling function previously described. This procedure is based on the assumption that the nuclear effects, encoded in the function $f$, are the same in the $\mathrm{QE}$ and inelastic regions and requires the knowledge of the elementary tensor $w_{\text {inel }}^{\mu \nu}$ across the full inelastic spectrum.

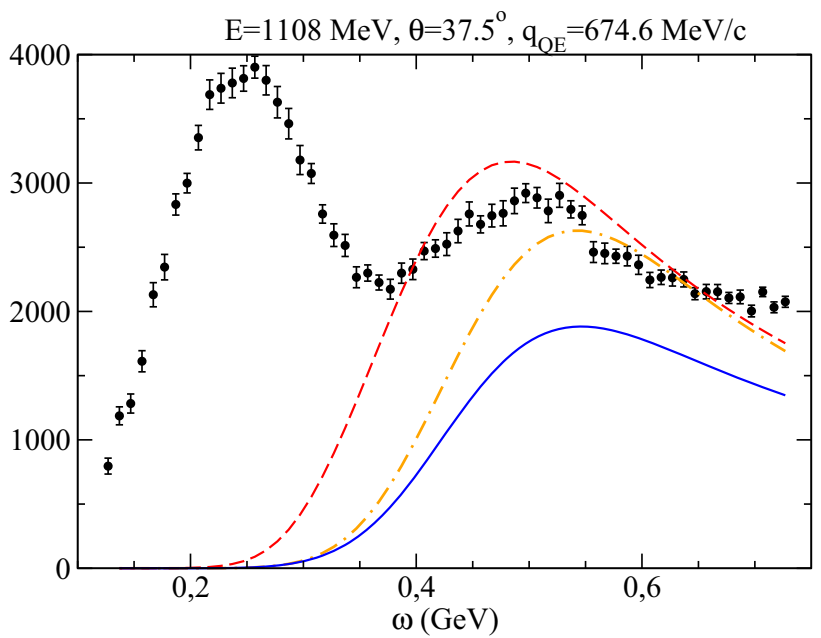

matics (incident electron beam and scattering angle) in terms of the energy transferred to the nucleus $(\omega)$. Experimental data taken from $[39,40]$. The $y$-axis represents $d^{2} \sigma / d \Omega / d \omega$ in $\mathrm{nb} / \mathrm{GeV} / \mathrm{sr}$. The value of $q$ at the $\mathrm{QE}$ peak $\left(q_{Q E}\right)$ is shown as reference. Figure adapted from [41]

The SuSAv2 inelastic model has been applied to the electron scattering case using phenomenological fits of the single-nucleon inelastic structure functions $w_{1}$ and $w_{2}$ extracted from e-p and e-d data $[33,34]$. In Fig. 2, a comparison of the SuSAv2-inelastic model with ${ }^{12} \mathrm{C}\left(e, e^{\prime}\right)$ data using different inelastic structure functions shows a preference for the Bosted-Christy parametrization $[33,34]$. In general, the SuSAv2 model (QE+inelastic) provides a very good description of data for very different kinematics once meson exchange currents (MEC) are also incorporated, denoted as SuSAv2MEC (see Sect. 2.4, [17,42,43]). The inclusion of the full inelastic spectrum in the SuSAv2 model for weak interactions is still in progress.

An alternative approach to the study of the resonant pion production was taken in Refs. $[15,44,45]$, where a scaling function to be used in the $\Delta$ resonance region, different from the quasielastic one, was extracted from electron scattering data and multiplied by the appropriate weak $N \rightarrow \Delta$ transition form factors to get the neutrino-nucleus cross section in this region. This method provides a phenomenological description valid at transferred energies below the $\Delta$ peak, while at higher $\omega$ it fails due to the opening of other inelastic channels. Some results corresponding to this method, referred to as SuSA- $\Delta$ approach, will be shown in Sect. 4.

\subsection{Relativistic model for CC MEC and 2p2h responses}

Multinucleon knockout processes give a non-negligible contribution to the inclusive neutrino cross section for the intermediate energies involved in the experiments [17,46-49]. In Ref. [50] we developed a model of twoparticle two-hole excitations of the RFG induced by 
Fig. 3 Feynman diagrams for the electroweak MEC

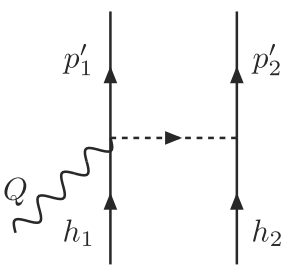

(a)

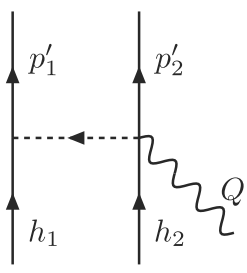

(b)

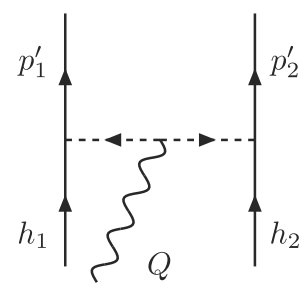

(c)

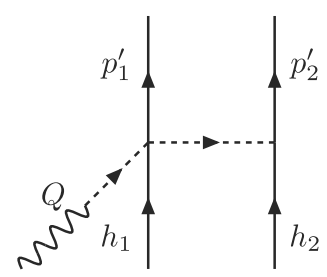

(d)

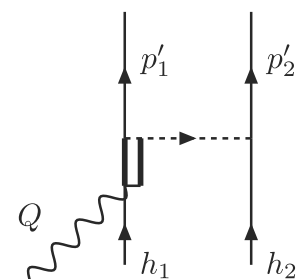

(f)

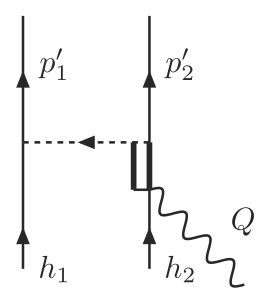

(g)

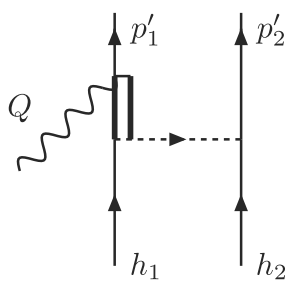

(h)

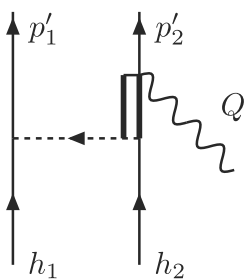

(i) weak meson-exchange currents for inclusive CC neutrino scattering. The model is fully relativistic and includes the diagrams of Fig. 3, involving one-pion exchange and $\Delta$ excitation, taken from the pion production model of [51].

The $2 \mathrm{p} 2 \mathrm{~h}$ matrix element of MEC depends on the momenta, spin and isospin coordinates $\left(1^{\prime}, 2^{\prime} ; 1,2\right) \equiv$ $\left(\mathbf{p}_{1}^{\prime} s_{1}^{\prime} t_{1}^{\prime}, \mathbf{p}_{2}^{\prime} s_{2}^{\prime} t_{2}^{\prime} ; \mathbf{h}_{1} s_{1} t_{1}, \mathbf{h}_{2} s_{2} t_{2}\right)$ of the two holes, $\mathbf{h}_{1}, \mathbf{h}_{2}$, and the two particles, $\mathbf{p}_{1}^{\prime}, \mathbf{p}_{2}^{\prime}$. It is the sum of four contributions

$$
\begin{aligned}
j^{\mu}\left(1^{\prime}, 2^{\prime} ; 1,2\right) & \equiv j^{\mu}\left(\mathbf{p}_{1}^{\prime} s_{1}^{\prime} t_{1}^{\prime}, \mathbf{p}_{2}^{\prime} s_{2}^{\prime} t_{2}^{\prime} ; \mathbf{h}_{1} s_{1} t_{1}, \mathbf{h}_{2} s_{2} t_{2}\right) \\
& =j_{\text {sea }}^{\mu}+j_{\pi}^{\mu}+j_{\text {pole }}^{\mu}+j_{\Delta}^{\mu},
\end{aligned}
$$

corresponding in Fig. 3 to the seagull (diagrams a,b), pion in flight (c), pion-pole (d,e) and $\Delta(1232)$ excitation (f,g,h,i). Their explicit expressions are given in Ref. [50].

The inclusive hadronic tensor in the $2 \mathrm{p}-2 \mathrm{~h}$ channel is computed by integration over all the $2 \mathrm{p}-2 \mathrm{~h}$ excitations of the RFG. Momentum conservation enforces $\mathbf{p}_{\mathbf{2}}^{\prime}=$ $\mathbf{h}_{\mathbf{1}}+\mathbf{h}_{\mathbf{2}}+\mathbf{q}-\mathbf{p}_{\mathbf{1}}^{\prime}$. Hence

$$
\begin{aligned}
W_{2 \mathrm{p} 2 \mathrm{~h}}^{\mu \nu}= & \frac{V}{(2 \pi)^{9}} \int d^{3} p_{1}^{\prime} d^{3} h_{1} d^{3} h_{2} \frac{m_{N}^{4}}{E_{1} E_{2} E_{1}^{\prime} E_{2}^{\prime}} \\
& w^{\mu \nu}\left(\mathbf{p}_{1}^{\prime}, \mathbf{p}_{2}^{\prime} ; \mathbf{h}_{1}, \mathbf{h}_{2}\right) \Theta\left(p_{1}^{\prime}, h_{1}\right) \Theta\left(p_{2}^{\prime}, h_{2}\right) \\
& \delta\left(E_{1}^{\prime}+E_{2}^{\prime}-E_{1}-E_{2}-\omega\right),
\end{aligned}
$$

where $\Theta\left(p^{\prime}, h\right) \equiv \theta\left(p^{\prime}-k_{F}\right) \theta\left(k_{F}-h\right)$. The tensor inside the integral is

$$
\begin{aligned}
& w^{\mu \nu}\left(\mathbf{p}_{1}^{\prime}, \mathbf{p}_{2}^{\prime} ; \mathbf{h}_{1}, \mathbf{h}_{2}\right) \\
& \quad \equiv \frac{1}{4} \sum_{s_{1} s_{2} s_{1}^{\prime} s_{2}^{\prime}} \sum_{t_{1} t_{2} t_{1}^{\prime} t_{2}^{\prime}} j^{\mu}\left(1^{\prime}, 2^{\prime} ; 1,2\right)_{A}^{*} j^{\nu}\left(1^{\prime}, 2^{\prime} ; 1,2\right)_{A},
\end{aligned}
$$

where $j^{\mu}\left(1^{\prime}, 2^{\prime}, 1,2\right)_{A}$ is the antisymetrized MEC matrix element

$$
j^{\mu}\left(1^{\prime}, 2^{\prime}, 1,2\right)_{A} \equiv j^{\mu}\left(1^{\prime}, 2^{\prime}, 1,2\right)-j^{\mu}\left(1^{\prime}, 2^{\prime}, 2,1\right) .
$$

The factor 1/4 in Eq. (17) accounts for the antisymmetry of the two-body wave function in isospin formalism, to avoid double counting in the number of final $2 \mathrm{p}-2 \mathrm{~h}$ states.

Due to azimuthal symmetry around the $z$-axis - in the $\mathbf{q}$ direction - we fix the azimuthal angle of particle 1 ' $\phi_{1}^{\prime}=0$, and multiply by a factor $2 \pi$. The energy deltafunction enables integrating over $p_{1}^{\prime}$. Then Eq. (16) is reduced to a seven dimensions integral that is computed numerically $[52,53]$. The Dirac matrix elements of the currents are also computed numerically.

The $2 \mathrm{p}-2 \mathrm{~h}$ inclusive cross section requires one to compute the five weak response functions, $R^{C C, C L, L L, T, T^{\prime}}$, 
for $\left(\nu_{\mu}, \mu^{-}\right)$. All of these responses were computed and analyzed in Ref. [50]. The five response functions have been parametrized in the kinematic range $100<q<$ 2000 [17]; the parametrization is convenient because in neutrino scattering there is an additional integration over the incident neutrino flux. This parametrization has been implemented in the Monte Carlo event generator GENIE $[43,54]$.

Using the parametrization of Ref. [17] does not allow one to modify the internal parameters of the MEC model. This is why we have developed approximations to the $2 \mathrm{p} 2 \mathrm{~h}$ responses to speed up the calculation. Specifically, in the so-called frozen nucleon approximation we set the momenta of the two holes $h_{1}=h_{2}=0$, allowing us to integrate over the initial states analytically [55]. In the modified convolution approximation (MCA) [56] we write the $2 \mathrm{p} 2 \mathrm{~h}$ responses as a convolution of two $1 \mathrm{p}-1 \mathrm{~h}$ responses multiplied by the elementary hadronic tensor $w^{\mu \nu}\left(\mathbf{p}_{1}^{\prime}, \mathbf{p}_{2}^{\prime} ; \mathbf{h}_{1}, \mathbf{h}_{2}\right)$ evaluated for convenient averaged values of $\mathbf{h}_{1}$ and $\mathbf{h}_{2}$. The resulting MCA responses are a good approximation to the exact result with $4 \mathrm{D}$ integration only over the momentum and energy communicated to one of the nucleons.

\subsection{Validation of the SuSAv2 model versus electron scattering data}

The model introduced in the previous sections allows one to describe inclusive lepton-nucleus scattering in the kinematic region including the quasielastic reaction, the excitation of $2 \mathrm{p} 2 \mathrm{~h}$ states and the inelastic spectrum. Validation against the large amount of existing highprecision electron scattering data is a necessary test to be performed before using nuclear models in the analysis of neutrino oscillation experiments. Such tests have been successfully carried out for the SuSAv2 model in a very wide range of kinematics from low/intermediate energies up to the highly inelastic regime. Only at very low energy and momentum transfers $(q<300 \mathrm{MeV} / \mathrm{c}$ and $\omega<50 \mathrm{MeV}$ ) does the model fail to reproduce the data, as do all models based on the impulse approximation, which is clearly not appropriate in the low-energy regime. SuSAv2 predictions have been extensively compared with the available $\left(e, e^{\prime}\right)$ data on carbon and oxygen in Refs. $[29,42]$ and with the more recent JLab data on argon and titanium in Ref. [57]. Here we just show a few representative examples, choosing the kinematics of particular interest for ongoing neutrino experiments.

In Fig. 4 (top left panel), the double differential ${ }^{12} \mathrm{C}\left(e, e^{\prime}\right)$ cross section is shown as a function of the energy transfer $\omega$, for an incident electron beam of energy $E$ and scattering angle $\theta$, corresponding to the momentum transfer $q_{\mathrm{QE}}$ at the quasielastic peak. The separate $\mathrm{QE}, 2 \mathrm{p} 2 \mathrm{~h}$ and inelastic contributions are shown. The agreement with the data is excellent across the full spectrum. Note that $2 \mathrm{p} 2 \mathrm{~h}$ excitations are essential to describe the "dip" region between the QE and $\Delta$ peaks.

An important feature of the SuSAv2 model, based on the RMF theory, is its capability to reproduce not only the $\left(e, e^{\prime}\right)$ cross sections but also the separated longitudinal and transverse responses, as shown in the top right panel of Fig. 4, where $R_{L}$ (solid) and $R_{T}$ (dashed) are plotted versus $\omega$ for $q=570 \mathrm{MeV} / \mathrm{c}$, together with the separate $\mathrm{QE}, 2 \mathrm{p} 2 \mathrm{~h}$ and inelastic contributions. Note that while the longitudinal response is almost purely quasielastic, with a small $2 \mathrm{p} 2 \mathrm{~h}$ contributions arising from relativistic corrections, the transverse response receives contributions from all the three processes, which sizeably overlap at these kinematics.

In Fig. 4 (bottom panels), the same kind of comparison is shown for the oxygen (left) and argon (right) nuclei, showing that the superscaling approach allows for a consistent description of different nuclei.

As already mentioned, electron scattering is an important test for the description of the nuclear response in a wide kinematical region. Such a detailed comparison with data is not possible for neutrino-nucleus scattering because the energy and momentum transfers are not experimentally known and because the large experimental errors do not allow for a clear discrimination between different models. The excellent agreement of the SuSAv2 predictions with electron scattering data proves that the model can be safely used to describe also neutrino-nucleus scattering, where the leptonic tensor is different but the nuclear dynamics, contained in the hadronic tensor, is the same as in charged-lepton scattering.

Before showing the comparison with neutrino data, in the next section we address the study of semi-inclusive electron scattering.

\section{Semi-inclusive electron-nucleus scattering}

A pivotal difference between electron and neutrino scattering experiments is that the neutrino energy is known only as a broad distribution, while the energy in electron beams is typically defined with high precision. Hence, in electron scattering experiments by, for instance, detecting one nucleon in coincidence with the scattered electron, the hadronic final state is completely determined if one restricts one's attention to excitation energies of the residual system below the two-nucleon emission threshold. On the contrary, in the neutrino case, even in scenarios in which one or a few hadrons are detected in coincidence with the scattered lepton, there is no way of fully determining the hadronic final state because the beam energy and consequently the excitation energy of the residual system is unknown. This means that one needs to 'integrate' for all possible final states compatible with the given kinematics. Formally, this integration is equivalent to the integration over the neutrino flux (or flux folding procedure) introduced in Sect. 2.1.

The semi-inclusive process for CCQE reactions was recently discussed in Refs. [60,61].

To obtain a somewhat deeper understanding of how the inclusive cross sections and scaling emerge, in this section we briefly discuss semi-inclusive (coincidence) 

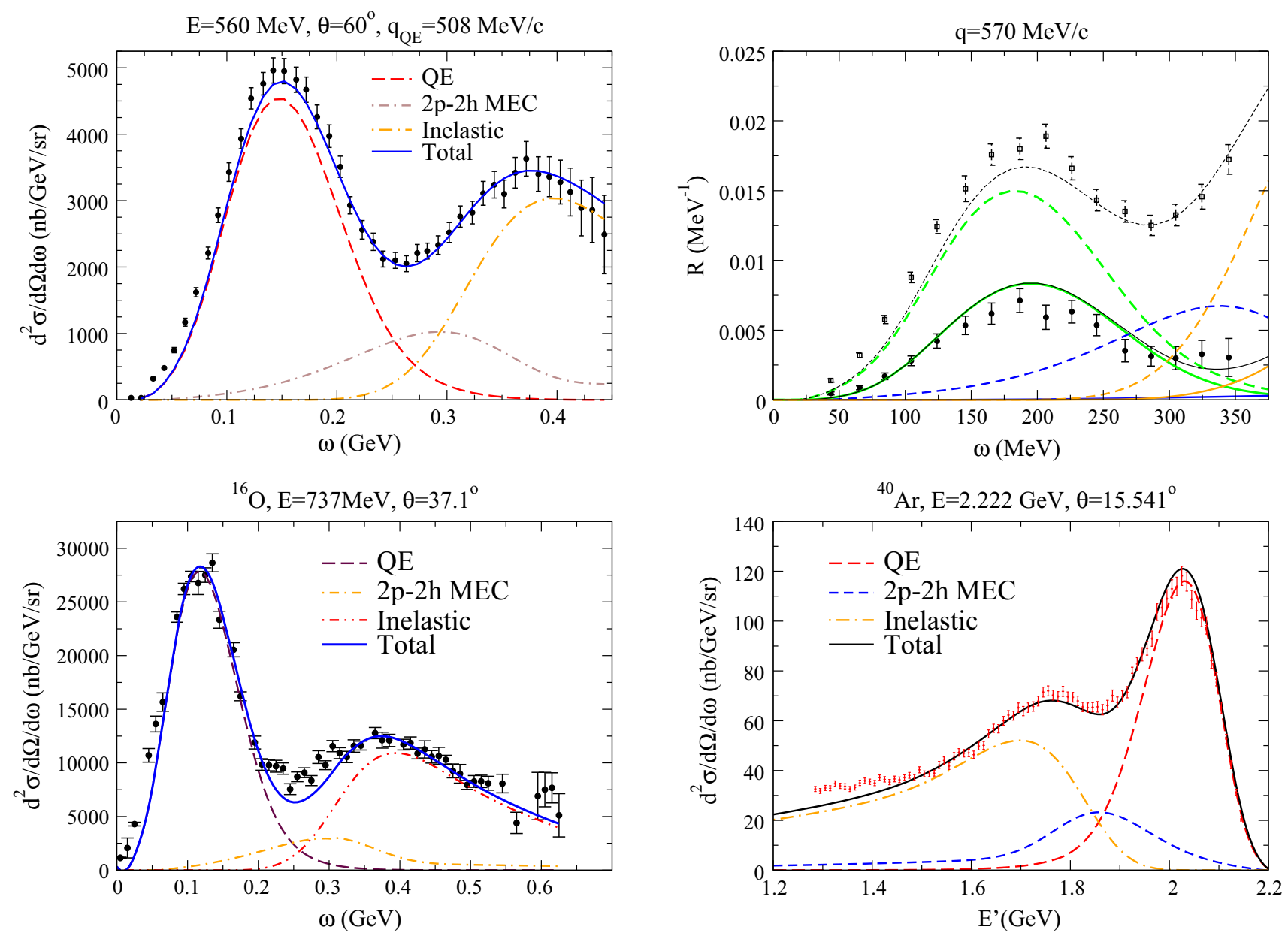

Fig. 4 Top left panel: The ${ }^{12} \mathrm{C}\left(e, e^{\prime}\right)$ cross sections from Refs. $[39,40]$ compared with SuSAv2-MEC predictions. The separate QE, $2 \mathrm{p}-2 \mathrm{~h}$ and inelastic contributions are also shown. Figure adapted from Ref. [29]. Top right panel: ${ }^{12} \mathrm{C}\left(e, e^{\prime}\right)$ longitudinal (solid) and transverse (dashed) responses at $q=570 \mathrm{MeV} / \mathrm{c}$. QE, $2 \mathrm{p} 2 \mathrm{~h}$ and inelastic contributions are shown, respectively, as green, blue and orange

electron scattering, focusing on the reaction $\left(e, e^{\prime} N\right)$ in which the scattering electron and a nucleon are assumed to be detected in the final state. Then, in addition to the electron kinematical variables introduced above, we have an outgoing nucleon with 4-momentum $P_{N}^{\mu}=\left(E_{N}, \mathbf{p}_{N}\right)$ involving 3-momentum $\mathbf{p}_{N}$ and polar and azimuthal angles $\theta_{N}$ and $\phi_{N}$, respectively, together with energy $E_{N}=\sqrt{p_{N}^{2}+m_{N}^{2}}$. No other particles are assumed to be detected, although, depending on the specific kinematics, they must be present (see below). The magnitude of the nucleon's 3-momentum is given by $p_{N}=\left|\mathbf{p}_{N}\right|$. Apart from the detected nucleon, the final state contains an undetected hadronic system having missing 4-momentum $\left(E_{B}, \mathbf{p}_{B}\right)$, namely, a total energy of $E_{B}$ and a missing 3-momentum $\mathbf{p}_{B} \equiv \mathbf{p}_{m}$. In the following we shall assume that the detected nucleon is a proton. One then has

$$
\mathbf{p}_{m}=\mathbf{q}-\mathbf{p}_{N} .
$$

lines. The total response is shown by the black lines. Data from Ref. [26]. Figure adapted from Refs. [18,41]. Bottom panels: The $\left(e, e^{\prime}\right)$ cross section (left) for ${ }^{16} \mathrm{O}$ from Ref. [58] and for ${ }^{40} \mathrm{Ar}$ (right) from Ref. [59] compared with the SuSAv2-MEC model. The separate QE, 2p-2h and inelastic contributions are also displayed. Figures adapted from Refs. [42] and [57], respectively

The undetected hadronic system has invariant mass $M_{B}\left(M_{B}^{0}\right.$ at threshold with $\left.M_{B} \geq M_{B}^{0}\right)$ and total energy

$$
E_{B}=T_{B}+M_{B}=\sqrt{\left(M_{B}\right)^{2}+p_{m}^{2}},
$$

which defines the kinetic energy of the unobserved finalstate system, $T_{B}$. From Eq. (20) one has

$$
E_{B}=\varepsilon-\varepsilon^{\prime}-T_{N}+\left(M_{A}^{0}-m_{N}\right),
$$

where $M_{A}^{0}$ is the target ground-state mass and $T_{N}=$ $E_{N}-m_{N}$ is the kinetic energy of the detected nucleon. This leads to the following expression for the so-called missing-energy,

$$
E_{m}=\varepsilon-\varepsilon^{\prime}-T_{N}-T_{B}
$$


Defining the excitation energy of the residual system

$$
\mathcal{E}=E_{B}-E_{B}^{0} \simeq M_{B}-M_{B}^{0},
$$

where the (typically very small) recoil kinetic energy difference has been neglected, we have

$$
E_{m}=\mathcal{E}+E_{s},
$$

where $E_{s}=M_{B}^{0}+m_{N}-M_{A}^{0}$ is the separation energy.

The magnitude of the missing-momentum $p_{m}$ is given by

$$
\begin{aligned}
p_{m}= & {\left[k^{2}+k^{\prime 2}+p_{N}^{2}-2 k k^{\prime} \cos \theta_{l}-2 k p_{N} \cos \theta_{N}\right.} \\
& \left.+2 k^{\prime} p_{N}\left(\cos \theta_{l} \cos \theta_{N}+\sin \theta_{l} \sin \theta_{N} \cos \phi_{N}\right)\right]^{\frac{1}{2}} .
\end{aligned}
$$

Depending on the value of the missing-energy, the residual system may be the daughter nucleus in its ground state (this defines the threshold for the reaction to become possible); or it may be in a discrete excited state (lying below the threshold where a second nucleon can be ejected), and, although they de-excite by $\gamma$-decay, that process is slow on the nuclear timescale and thus these states may be treated effectively as stationary states. Then, at a well-defined threshold a second nucleon must be emitted (this is not optional: there are no nuclear states involving one nucleon and a residual bound nucleus above this point). As $E_{m}$ continues to increase more and more particles enter in the final state in addition to the one special nucleon that is assumed to be detected. At even larger missing energy (roughly $140 \mathrm{MeV}$ ) pion production becomes possible (still with the lepton and one nucleon assumed to be detected).

From Eqs. (22-25) it is clear that for fixed values of the observable parameters the values of $E_{m}$ and $p_{m}$ are determined for each value of $\varepsilon$. In Fig. 5 trajectories are shown for selected kinematics, namely, $\varepsilon^{\prime}=3800 \mathrm{MeV}$, $\theta_{l}=7^{\circ}, T_{N}=140 \mathrm{MeV}, \phi_{N}=180^{\circ}$, representing a "typical" situation. What is varied here is the polar angle for the detected proton, $\theta_{N}$. As stated above, as one goes along a given trajectory the electron energy $\varepsilon$ that determines where on the trajectory one finds oneself must vary. The lower boundary defines the threshold for the semi-inclusive reaction to occur. In effect, each event where an electron and a proton are detected in coincidence corresponds to a specific trajectory and point on that trajectory.

All realistic representations of the missing-energy and missing-momentum distribution of the nucleons in the target nuclei, the so-called spectral function, share essentially the same ingredients, that is, a mean field part that describes the shells, with their widths somehow adjusted to the available experimental information, and a background contribution corresponding to the high missing-energy and high missing-momentum part of the spectrum, that is associated to short-range correlations. In this work, we will consider the spectral

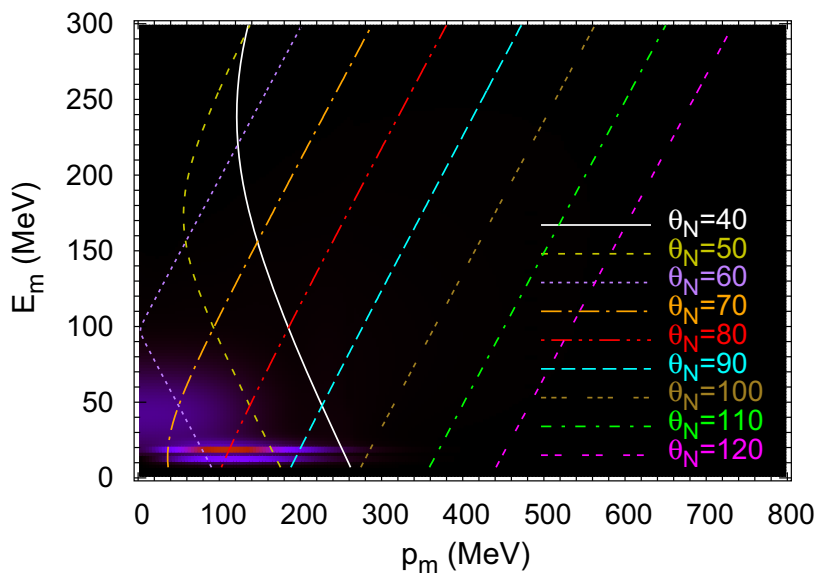

Fig. 5 The $E_{m}-p_{m}$ trajectories are shown for selected "typical" kinematics: $\varepsilon^{\prime}=3800 \mathrm{MeV}, \theta_{l}=7^{\circ}, T_{N}=140$ $\mathrm{MeV}, \phi_{N}=180^{\circ}$ for the reaction ${ }^{16} \mathrm{O}\left(e, e^{\prime} p\right)$. Each line corresponds to a different value of the proton scattering angle $\theta_{N}$ (in degrees). Here, we plot the Rome spectral function as a background to allow one to easily identify the different regions of the spectral function that are crossed by the trajectories

function of Refs. [62,63], labeled for brevity as "Rome spectral function". Though other models are available in the literature, e.g., Refs. [64,65], all of them have been constrained by electron scattering experiments, and hence they will provide similar results. When going along a given trajectory in Fig. 5, one sees a striking pattern in the behavior one should expect. The strength is extremely localized. The largest concentration occurs where the $p$-shells are located (at around $E_{m}=20$ $\mathrm{MeV}$ ), with less strength where the broad $s$-shell is located (at around $E_{m}=50 \mathrm{MeV}$ ); at still larger values of $E_{m}$ (and $p_{m}$ ) the spectral function does have some strength, although it is spread over a wide region in the $E_{m}-p_{m}$ plane and is too small to be seen in this representation. Furthermore, we note that pion production cannot occur until one reaches $E_{m} \sim m_{\pi}$ and that it is not appreciable until $E_{m} \sim m_{\Delta}-m_{N} \sim 300 \mathrm{MeV}$.

We notice that in the case of the CCQE neutrino interaction, studied in Ref. [61], one gets a $E_{m}-p_{m}$ trajectory plot that is almost identical to the one in Fig. 5. The reason is that the muon mass does not play a strong role in these trajectories, especially for the kinematics shown here for which $\varepsilon^{\prime}$ is much larger than the muon mass.

In passing we note that, while other choices of two variables to replace $E_{m}$ and $p_{m}$ can of course be made, the generic behavior seen here strongly suggests that the present choice is a good one and that other choices may not reflect the highly localized nature of the nuclear response.

In Fig. 6, we represent the sixfold differential cross section for fixed electron and proton kinematics as a function of the missing-energy. The model used was PWIA together with the Rome spectral function [62, 63], as described, e.g., in Refs. [60,66]. Equation (22) 
(a)

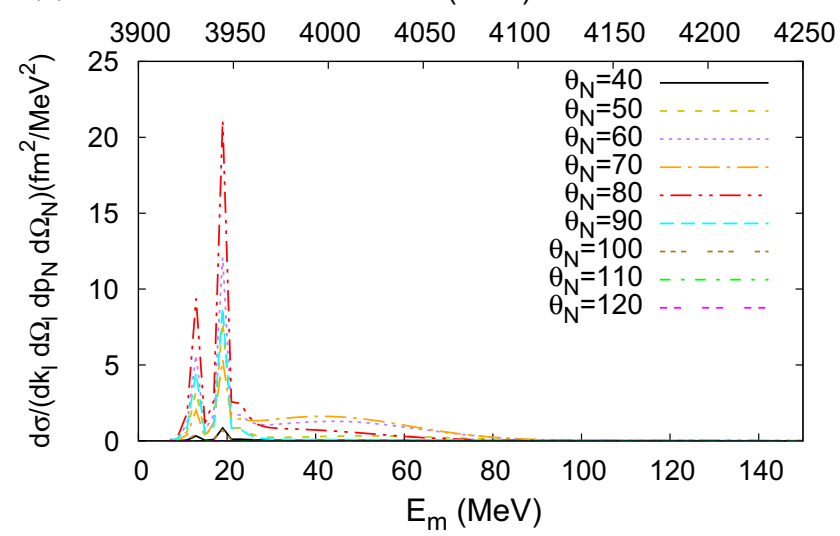

Fig. 6 The sixfold ${ }^{16} \mathrm{O}\left(e, e^{\prime} p\right)$ differential cross section as a function of the missing-energy $E_{m}$ (lower $x$-axis) and the neutrino energy (upper $x$-axis) on linear (a) and semi-log

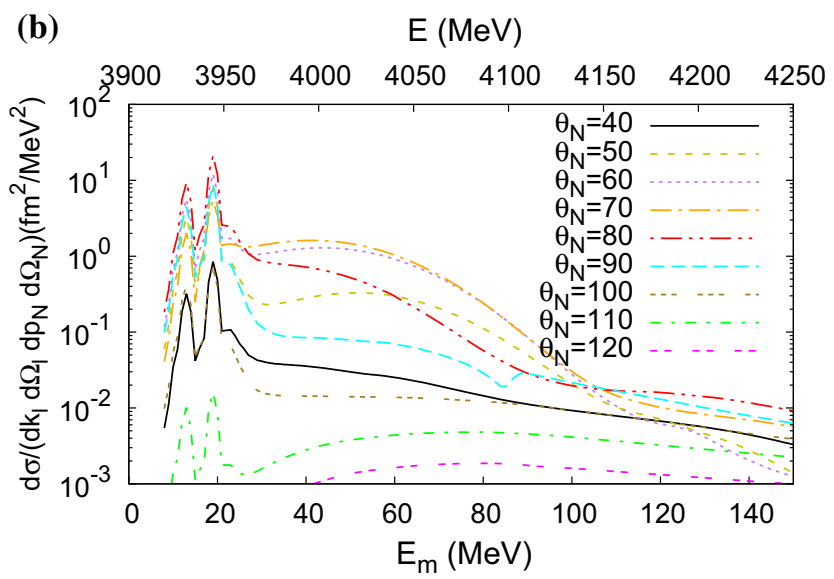

(b) scales. The electron and proton variables are fixed to: $\varepsilon^{\prime}=3800 \mathrm{MeV}, \theta_{l}=7^{\circ}, T_{N}=140 \mathrm{MeV}, \phi_{N}=180^{\circ}$, as in Fig. 5
Fig. 7 Excitation energy $\mathcal{E}=E_{m}-E_{s}$ versus missing momentum $p=p_{m}$. The shaded area represents the kinematically allowed region for $y<0$ (left panel) and $y>0$ (right panel)
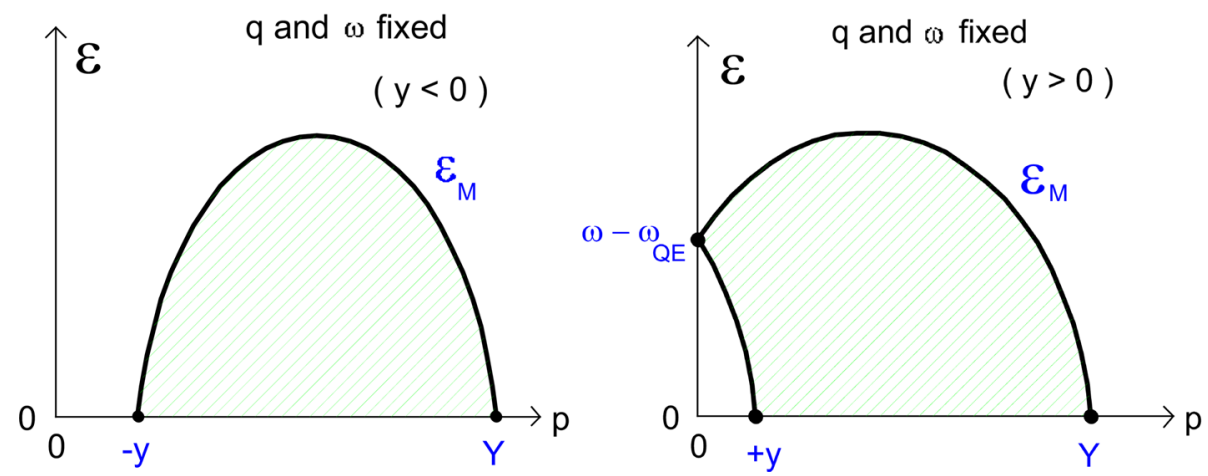

tells us that for fixed nucleon and final lepton energies, if one neglects the kinetic energy of the residual system $T_{B}$, which under typical QE conditions is always very small, then one finds a one-to-one linear relation between the initial lepton energy and the missing energy. Thus, in Fig. 6 the beam energy is shown as a second $x$-axis. By varying the angle $\theta_{N}$, the cross section changes its magnitude and its shape. One observes two prominent peaks corresponding to the $p$ shells, a wide bump for the $s$-shell and a background that extends up to high missing-energies. Clearly, as expected, the $p$-shell strength is largest, the $s$-shell strength is smaller and the high- $E_{m}$ strength is completely negligible, being down by several orders of magnitude. By examining these results in the light of the trajectories shown in Fig. 5 we see that the general behavior we expect to occur is borne out. For example, the trajectories for $\theta_{N}=60^{\circ}$ and $80^{\circ}$ both pass though the $p$-shell region near its peak. However, one trajectory intersects the $s$-shell region more than the other one does and this results in relatively different amounts from the $s$-shell compared with the $p$-shell. Or, large values of $\theta_{N}$ correspond to very small cross sections, as they should, since neither the $p$ - nor $s$-shell regions are crossed.

We do have some knowledge about this generic behavior of the distribution of strength from inclusive electron scattering, $\left(e, e^{\prime}\right)$. Inclusive scattering corre- sponds to performing integrals over specific regions in the $E_{m}-p_{m}$ plane $[12,18,67]$. In Fig. 7 , we show the typical situation for kinematics at values of $q$ and $\omega$ where one is below the QE peak $(y<0$, left panel) or above the peak $(y>0$, right panel). In each case the inclusive QE cross section is obtained by integrating the semi-inclusive $\left(e, e^{\prime} N\right)$ cross sections over the shaded regions. Accordingly one can see that the two classes of cross section are intrinsically related. Clearly, if one had complete knowledge of the semi-inclusive response for a wide range of kinematics then the integrations could be performed to yield the inclusive response. Unfortunately this is not the case. Note that having a model for the inclusive cross section (the total hadronic cross section) does not mean that such a model will be valid for the semi-inclusive cross section (which constitutes the integrand of the former). An example of this is the RFG model which is not unreasonable for the total (inclusive) QE cross section, but is poor for the semi-inclusive response.

An example is that of the model used for the semiinclusive response discussed above. If employed for the inclusive cross section one finds for ${ }^{16} \mathrm{O}$ that somewhat over $50 \%$ of the inclusive cross section stems from the $p$-shells, about 25\% comes from the $s$-shell region and the rest comes from a broad region at higher missingenergy. This strength at higher missing energy is partially responsible for the asymmetry found in the scal- 

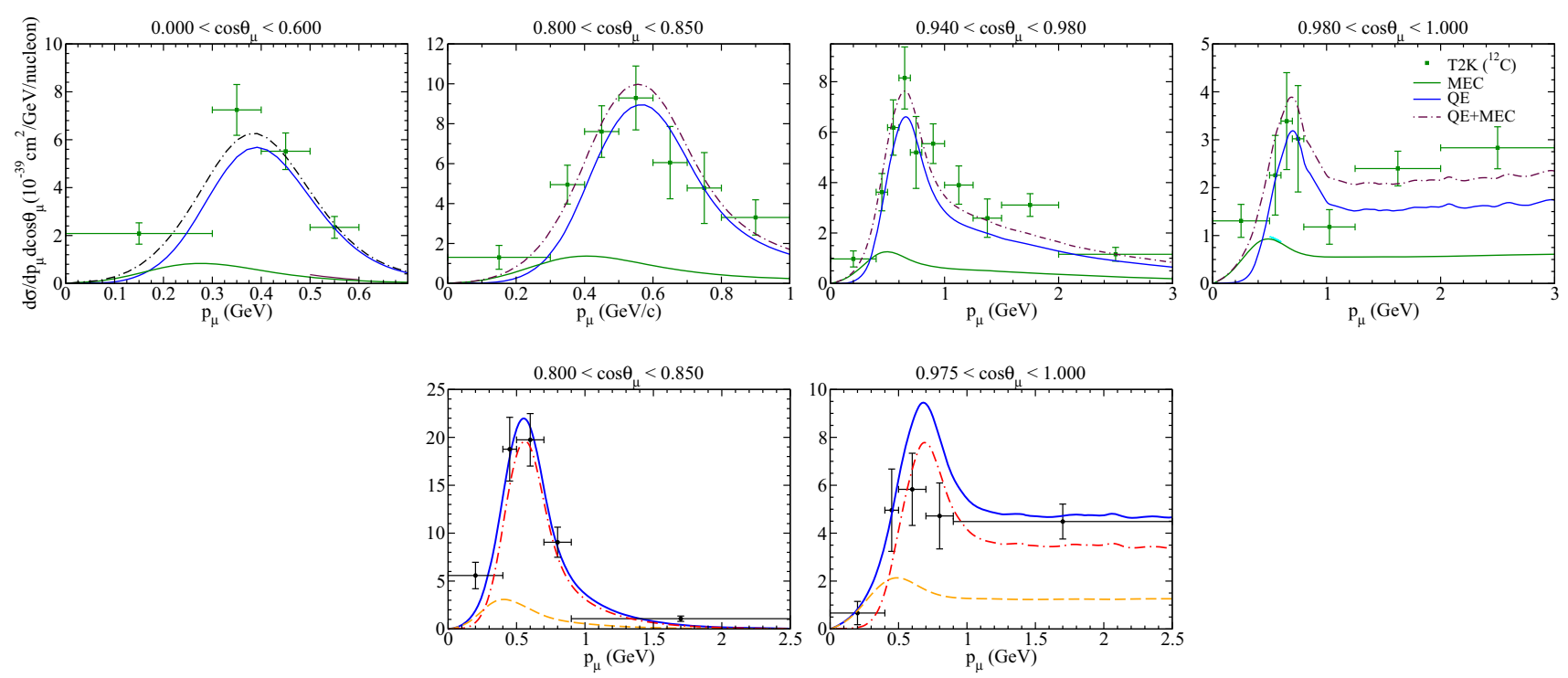

Fig. 8 Comparison of the SuSAv2-MEC model with the T2K flux-integrated CCQE and $2 \mathrm{p} 2 \mathrm{~h}$ double-differential cross section for neutrino scattering on ${ }^{12} \mathrm{C}$ (top panels) and ${ }^{16} \mathrm{O}$ (bottom panels) in units of $10^{-39} \mathrm{~cm}^{2} / \mathrm{GeV}$ per

nucleon (for carbon) and per nucleon target (for oxygen). The $\mathrm{CC} 0 \pi$ T2K data are from Refs. [68] and [69]. Figures adapted from Ref. [42]

ing functions. Note that inclusive scattering at high momentum transfers and hence the scaling functions involve broad integrals, whereas semi-inclusive scattering at fixed final-state electron and nucleon momenta involves a trajectory in the $E_{m}-p_{m}$ plane as the beam energy is varied. In detailed analyses one finds much more strength in that case coming from the valence knockout region, with much less arising from the high$E_{m}$ region. This has consequences for $\mathrm{CC} \nu$ reactions as discussed in Ref. [61].

\section{Selected results}

In Sect. 2.4, the validation of the SuSAv2-MEC model based on the RMF theory with $\left(e, e^{\prime}\right)$ data has been proven as a solid benchmark to assess the validity of a nuclear model before its application to neutrino reactions. Here, we show the capability of the SuSAv2-MEC model to describe a wide range of kinematics of interest for neutrino oscillation experiments. In particular, we focus on the comparisons of the SuSAv2-MEC model with charged-current neutrino cross sections from different experiments, in particular T2K and MINERvA. While T2K is an oscillation experiment, MINERvA is specifically dedicated to high-precision measurements of neutrino cross sections on various nuclei, providing input for oscillation studies.

Our analysis is mainly related to charged-current quasielastic-like events, also called $\mathrm{CC} 0 \pi$, which are characterized by having no pions detected in the final state and are dominated by the QE and $2 \mathrm{p} 2 \mathrm{~h}$ channels. Finally, we extend the analysis to cross section measurements where pion production is also included.
In Fig. 8, we show the comparison of the SuSAv2MEC model with $\mathrm{T} 2 \mathrm{~K} \mathrm{CC} 0 \pi$ double differential cross sections on ${ }^{12} \mathrm{C}$ (top panels) and ${ }^{16} \mathrm{O}$ (bottom panels). These data are compared with the $\mathrm{QE}$ and $2 \mathrm{p} 2 \mathrm{~h}$ MEC contributions showing an overall good agreement. Monte Carlo event generators also include other contributions that can mimic a CCQE-like event in the data analysis such as pion-absorption processes in the nucleus. Nevertheless, as it will be discussed later, these contributions are not particularly relevant at T2K kinematics. It is also important to note that the $\mathrm{QE}$ and $2 \mathrm{p} 2 \mathrm{~h}$ contributions in the SuSAv2-MEC model can be easily extrapolated from one nucleus to another by means of scaling rules, which are different for each nuclear regime. This is based on the assumption of $2 \mathrm{nd}-$ kind scaling, already mentioned in Sect. 2.2, and that has been proven in the SuSAv2-MEC approach for the $\mathrm{QE}$ and $2 \mathrm{p} 2 \mathrm{~h}$ channels $[57,70]$. It is worth commenting on the case of the most forward-angle region, associated with low kinematics, i.e., low energy and momentum transferred to the nucleus.

Within this region, the SuSAv2-MEC model slightly overestimates the $\mathrm{T} 2 \mathrm{~K}$ data for $\mathrm{C}$ and $\mathrm{O}$, being more noticeable in the latter case. This is mainly due to RMF scaling violations, related to low-energy nuclear effects and to different binding energies for each nucleus, which are not properly accounted for in the SuSAv2 approach. This limitation of the model has been solved in the recent ED-RMF approach [30,31] where the goodness of the RMF strong vector and scalar potentials at lowintermediate kinematics are present while also retaining the benefits of the SuSAv2 model description at larger kinematics, as described in Sect. 2.2. The differences introduced by this more accurate description of low-energy nuclear effects in the RMF (and ED- 

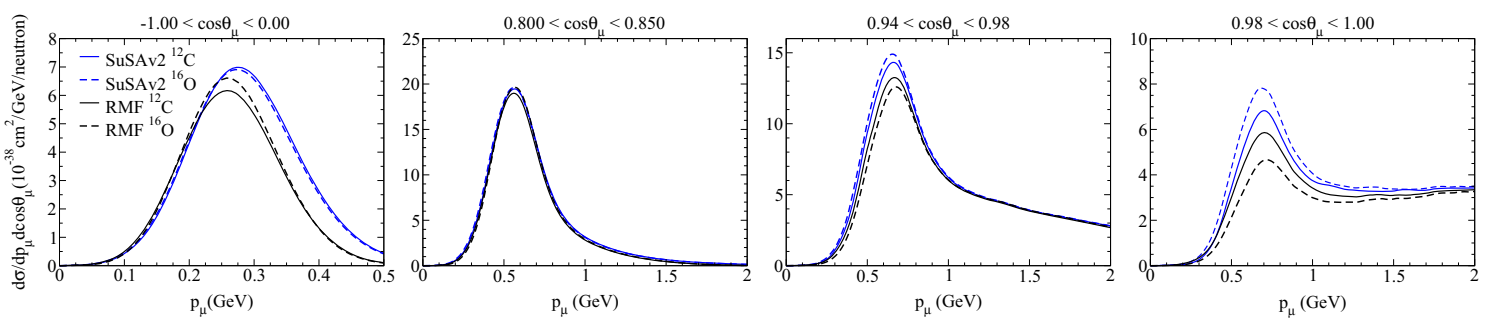

Fig. 9 Comparison of double differential cross sections on ${ }^{12} \mathrm{C}$ (solid lines) and ${ }^{16} \mathrm{O}$ (dashed lines) at $\mathrm{T} 2 \mathrm{~K}$ kinematics within the SuSAv2 (1p1h) and RMF models. Results are displayed from forward to backward angles

RMF) model can be observed in Fig. 9 where large discrepancies between oxygen and carbon predictions are observed between the RMF model and the SuSAv2MEC one at very forward angles, being smaller as we move to more backward kinematics. At the same time, these differences are more prominent in the case of oxygen. This is connected with the assumptions made in the SuSAv2 approach which is based on the RMF analysis on ${ }^{12} \mathrm{C}$ but on the application of scaling rules to describe other nuclear targets instead of relying on particular RMF predictions for each nucleus. Therefore, at kinematics where scaling violations are present, these differences, while noticeable in the nucleus of reference $\left({ }^{12} \mathrm{C}\right)$, can be even more important for other nuclear targets. On the contrary, SuSAv2 assumes that scaling works well even at low kinematics, thus implying minor differences between ${ }^{12} \mathrm{C}$ and ${ }^{16} \mathrm{O}$, mainly due to the scaling rule and differences in the energy shift introduced for each nucleus. This analysis is of relevance for $\mathrm{T} 2 \mathrm{~K}[71]$ where the accuracy of the $\mathrm{C}$ to $\mathrm{O}$ extrapolation plays an important role on the oscillation analysis and whose predictions have shown some discrepancies between $\mathrm{C}$ and $\mathrm{O}$ for very forward-going muons which may be explained within the RMF model. On the contrary, other theoretical nuclear models present in event generators exhibit smaller differences between these two targets [71] even at low kinematics.

The SuSAv2-MEC model predictions are also compared in Fig. 10 with the MINERvA CCQE-like double differential (anti)neutrino measurements on hydrocarbon $(\mathrm{CH})$ in terms of the transverse momentum of the outgoing muon (with respect to the antineutrino beam), in bins of the muon longitudinal momentum. Due to its relativistic nature, the SuSAv2-MEC model is well suited to describe these data $[43,72]$ where the mean neutrino energy is around $3.5 \mathrm{GeV}$. An overall good agreement is reached without resorting to any tuning or additional parameters. According to Refs. [43,72], the MINERvA "QE-like" cross sections entail, besides pure quasielastic contributions, events that have postFSI final states without mesons, prompt photons above nuclear de-excitation energies, heavy baryons, or protons above a kinetic energy threshold of $120 \mathrm{MeV}$, thus including zero-meson final states arising from resonant pion production followed by pion absorption in the nucleus and from multi-nucleon interactions. Apart from the "QE-like" points, the MINERvA "CCQE" signal is also shown, and corresponds to events initially generated in the GENIE event generator [74] as quasielastic (that is, no resonant or deep inelastic scatters, but including scatters from nucleons in correlated pairs with zero-meson final states), regardless of the final-state particles produced, thus including CCQE and $2 \mathrm{p} 2 \mathrm{~h}$ reactions. The difference between the two data sets is mainly related to pion production plus reabsorption and goes from $\sim 15$ to $\sim 5 \%$ depending on the kinematics. The present SuSAv2-MEC results do not include processes corresponding to these pion reabsorption processes inside the nucleus and the comparison should be done with the "CCQE" data rather than with the "QE-like" ones. A more detailed analysis of these results together with a $\chi^{2}$ test can be found in Ref. [43], where the SuSAv2 $\chi^{2}$ shows its compatibility with data and with the MINERvA/GENIE predictions. It is also important to note that due to MINERvA's acceptance, the muon scattering angle is limited to $\theta_{\mu}<20^{\circ}$ as well as the muon kinematics (1.5 $\left.\mathrm{GeV}<p_{\|}<15 \mathrm{GeV}, p_{T}<1.5 \mathrm{GeV}\right)$ in both experimental and theoretical results. This implies important phase-space restrictions for large energy and momentum transfer to the nuclear target and makes the available phase space not so different from the T2K one, as shown in Ref. [75].

The previous T2K and MINERvA results are only related to the final lepton kinematics, the so-called inclusive measurements. At this point, it is worth mentioning that other models [47, 48,76-96] have been also developed to address these CC inclusive neutrino interactions and, although similar agreement with data can be obtained, they are based on different assumptions about the nuclear properties and dynamics. Combined analyses of these models with more exclusive neutrino measurements where hadron kinematics and other nuclear effects can be analyzed in more detail would help to improve model selection for data analysis (see also the discussions in Sect. 3). The advantage of SuSAv2-MEC (and RMF) is that of being a fully relativistic model that has shown an overall good agreement with electron and neutrino scattering data and that can be extended without further approximations to the fullenergy range of interest for present and future neutrino experiments. For this reason, the SuSAv2-MEC model (1p1h and $2 \mathrm{p} 2 \mathrm{~h}$ ) has been recently implemented in the GENIE neutrino event generator [74] with the aim of improving the characterisation of the nuclear effects in neutrino cross section measurements and work is now in progress to implement this model together with the 

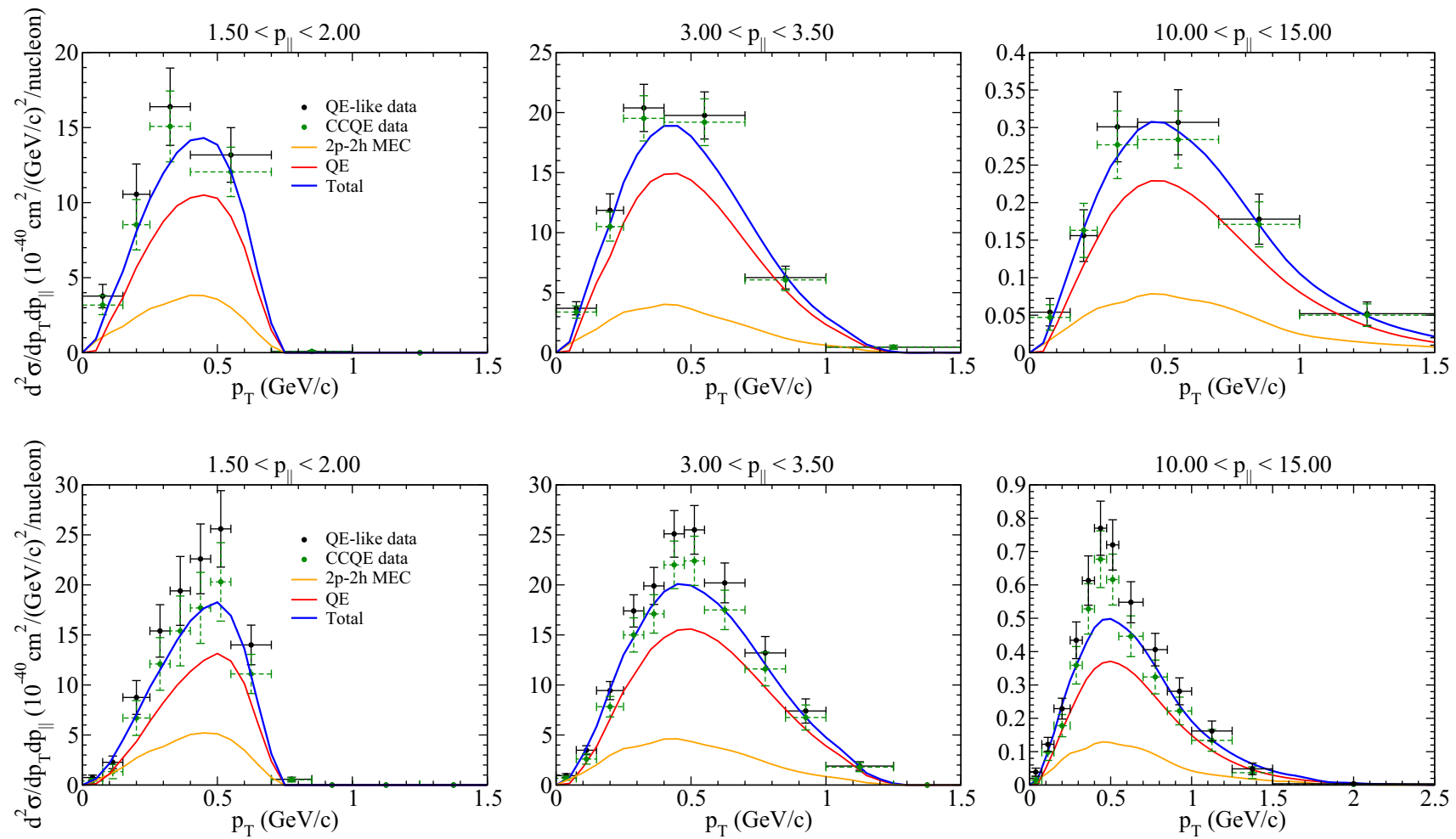

Fig. 10 The MINERvA "QE-like" and "CCQE" double differential cross sections for $\bar{\nu}_{\mu}$ (top panels) and $\nu_{\mu}$ (bottom panels) scattering on hydrocarbon versus the muon transverse momentum, in bins of the muon longitudinal momentum (in $\mathrm{GeV} / \mathrm{c}$ ). The curves represent the predic- tion of the SuSAv2+2p2h-MEC (blue) as well as the separate quasielastic (red) and $2 \mathrm{p} 2 \mathrm{~h}-\mathrm{MEC}$ (orange) channels. MINERvA data and experimental fluxes are from Refs. [72] and [73]. Figures adapted from Ref. [18,43]
ED-RMF one in the NEUT event generator [97] for its application on the $\mathrm{T} 2 \mathrm{~K}$ oscillation analysis.

To illustrate the implementation of the SuSAv2 model in GENIE, in Fig. 11 we show the T2K CC0 $\pi$ cross section with final protons of momentum lower than $500 \mathrm{MeV}$ [98]. The data are compared with the SuSAv2-1p1h, SuSAv2-2p2h and pion-absorption predictions from GENIE (see Ref. [54] for details). At proton momentum below $500 \mathrm{MeV} / \mathrm{c}$ a clear dominance of the SuSAv2-1p1h channel is observed. Note that SuSAv2-GENIE also shows an overestimation of data at very forward angles, as observed in Fig. 8, mainly due to low energy transfer scaling violations which are absent in the SuSAv2-model but present in the RMF theory. To analyze the low-energy nuclear effects and these scaling violations, we also show the comparison with the RMF model for the $1 \mathrm{p} 1 \mathrm{~h}$ channel together with the SuSAv2-2p2h and pion-absorption results from GENIE, noticing a remarkable improvement in the data comparison at very forward angles.

The accuracy and equivalency of the SuSAv2-MEC implementation in GENIE with respect to the original model has been shown in Ref. [54] for inclusive reactions. It is worth noticing that the SuSAv2 model is a purely inclusive model, that is, it only predicts lepton kinematics. Thus, its implementation in MC event generators has to rely on a factorization approach and on the nucleon momentum distribution information available in these generators from other approaches to produce predictions for hadron kinematics (see Ref. [54] for details). This limitation will be addressed in the forthcoming implementation of the ED-RMF model in event generators, which will be able to provide a consistent description of lepton and hadron kinematics in the final state together with an accurate description of the nuclear dynamics. In Ref. [61], the semi-inclusive cross sections has been studied in detail using different models and descriptions of FSI. In particular, the ED-RMF model has shown very similar results to the SuSAv2 for lepton kinematics on CCQE neutrino interactions [31]. A systematic study of neutrino semi-inclusive reactions within the ED-RMF model is in progress [99].

Due to aforementioned dominance of the $1 \mathrm{p} 1 \mathrm{~h}$ channel on $\mathrm{CC} 0 \pi$ interactions with low momentum protons observed in Fig. 11, it remains difficult to draw clear conclusions about the goodness of the $2 \mathrm{p} 2 \mathrm{~h}$ description.

Nevertheless, this can be explored further using more semi-inclusive measurements with measurements of proton and muon kinematics as shown in Fig. 12, where the SuSAv2-MEC model in GENIE is compared with the so-called single transverse variables (STV), and in particular with the transverse momentum imbalance, $\delta p_{T}$, defined in terms of the momentum imbalances between the outgoing muon and highest momen- 

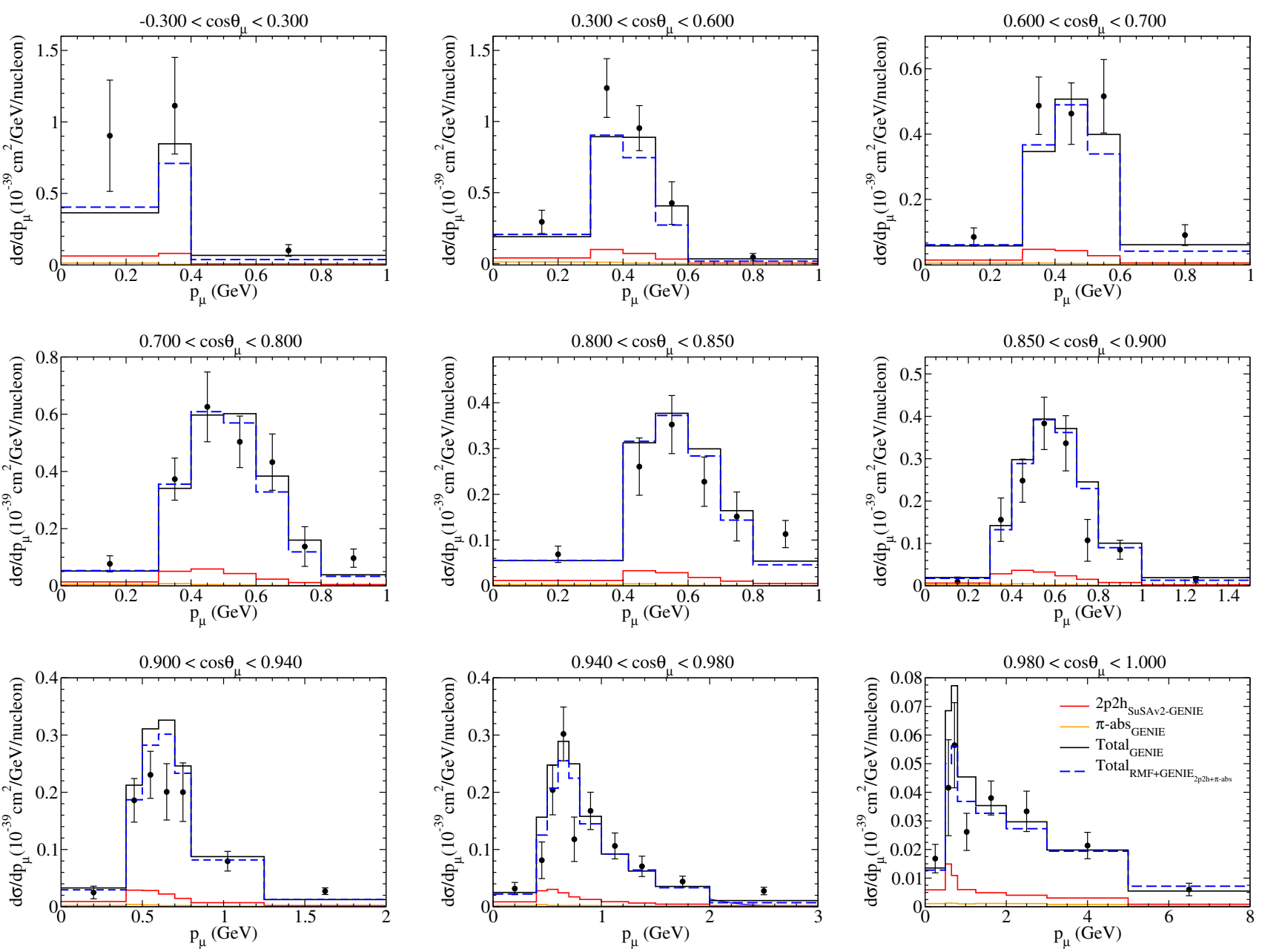

Fig. 11 Comparisons of data and model predictions for differential $\mathrm{CC} 0 \pi$ muon-neutrino cross sections on ${ }^{12} \mathrm{C}$ in the T2K neutrino beam as a function of the muon kinematics when there are no protons with momenta above 500 $\mathrm{MeV}$. Two 1p1h predictions are shown (one from RMF, the other from SuSAv2 implemented in GENIE), in addition to the SuSAv2 $2 \mathrm{p} 2 \mathrm{~h}$ and pion absorption contributions from
GENIE. The total contributions when using each of the two $1 \mathrm{p} 1 \mathrm{~h}$ models is also shown. Goodness of fit are calculated to be $\chi_{R M F}^{2}=171.87$ (59 bins) and $\chi_{S u S A}^{2}=168.92$ (60 bins), where the latter includes a single extra bin from - 1.0 to $0.3 \cos \theta$ (not shown). The data points are taken from Ref. [98]. Figure adapted from Ref. [54]

backs can be addressed in further works via the implementation of RMF models for the 1p1h channel and a full semi-inclusive $2 \mathrm{p} 2 \mathrm{~h}$ model. Apart from these limitations, it is worth noticing the almost perfect description of the shape of $\delta p_{T}$ in the right panel, improving the agreement reached by other descriptions also implemented in generators.

To conclude this section, we show the comparison of the SuSAv2-MEC model with measurements that also consider non-QE contributions, mainly pion production. This reaction channel is also of relevance for present and future neutrino oscillation experiments. Neutral current $\pi^{0}$ production constitutes an important background in the electron neutrino and antineutrino appearance analyses but also neutrino-induced pions emitted can mimic QE-like events if they are not properly detected or if they are absorbed due to FSI effects. Thus, the analysis and detection of these pion 


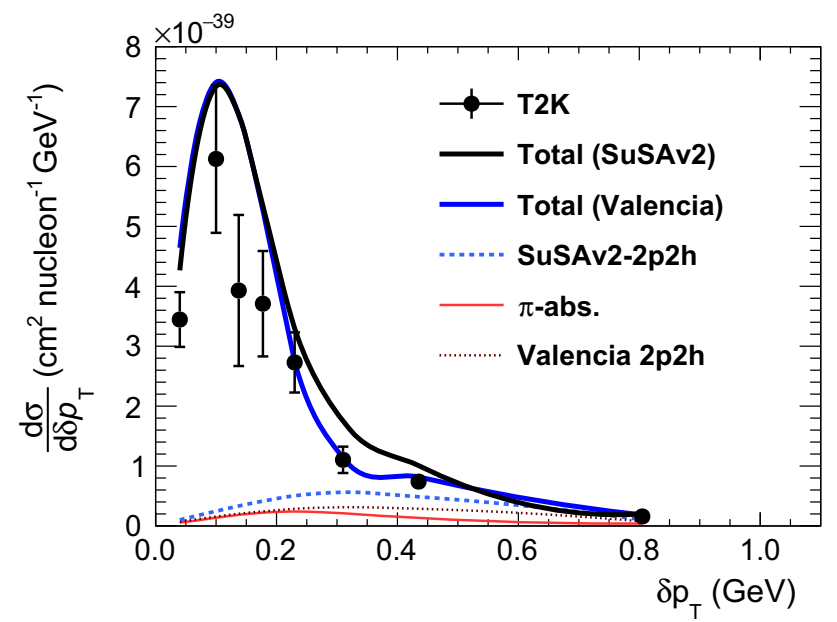

Fig. 12 The regularised $\mathrm{T} 2 \mathrm{~K}$ measurement of $\mathrm{CC} 0 \pi$ muon-neutrino cross sections on ${ }^{12} \mathrm{C}$ at $\mathrm{T} 2 \mathrm{~K}$ kinematics as a function of the Single Transverse Variable [100] $\delta p_{T}$ compared to predictions from the GENIE-implemented SuSAv2 and Valencia $1 \mathrm{p} 1 \mathrm{~h}+2 \mathrm{p} 2 \mathrm{~h}$ models, each of which is added to GENIE's pion absorption prediction. The total contri-

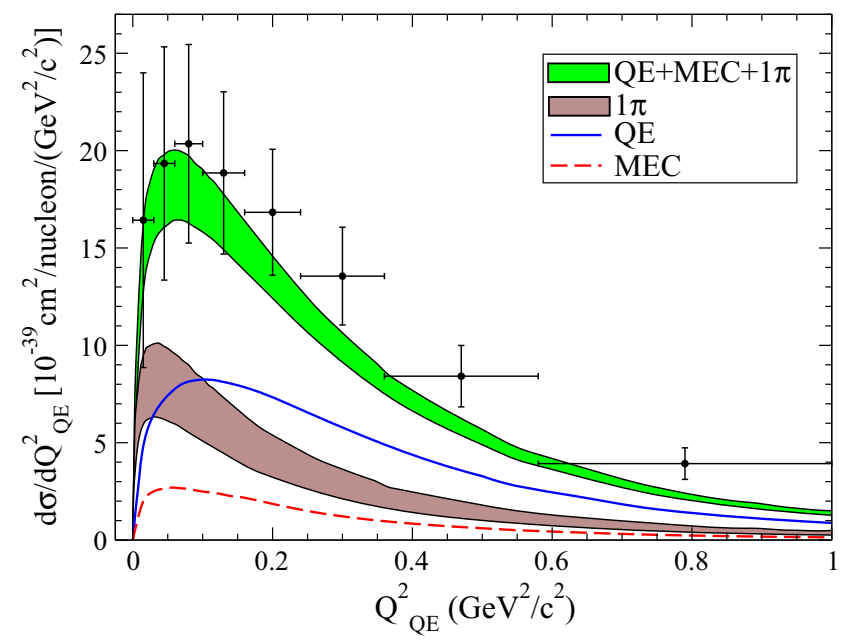

Fig. 13 (Left panel) We show the CC-inclusive T2K fluxfolded $\nu_{e^{-}}{ }^{12} \mathrm{C} Q_{Q E}^{2}$ differential cross section per nucleon. (Right panel) The CC $\nu_{\mu}$ total cross section on $\mathrm{C}_{8} \mathrm{H}_{8}$ is presented. Experimental data are from T2K [102] and

production events in coincidence with the final lepton and other hadrons is of paramount importance for the neutrino energy reconstruction.

To analyze the neutrino-induced pion production channel we make use of the phenomenological SuSA$\Delta$ approach which extends the superscaling arguments observed in the $\mathrm{QE}$ regime to the $\Delta$ resonance region (see Ref. [45] for details). In Fig. 13 (left panel) we show the $\nu_{e^{-}}{ }^{12} \mathrm{C}$ inclusive differential cross section averaged with the T2K flux versus the reconstructed fourmomentum transfer, $Q_{Q E}^{2}$, and in Fig. 13 (right panel) the $\nu_{\mu}$ total cross section on $\mathrm{C}_{8} \mathrm{H}_{8}$ target. Three contributions are shown in these plots, namely, the SuSAv2 QE and SuSAv2 2p2h-MEC channels together with the

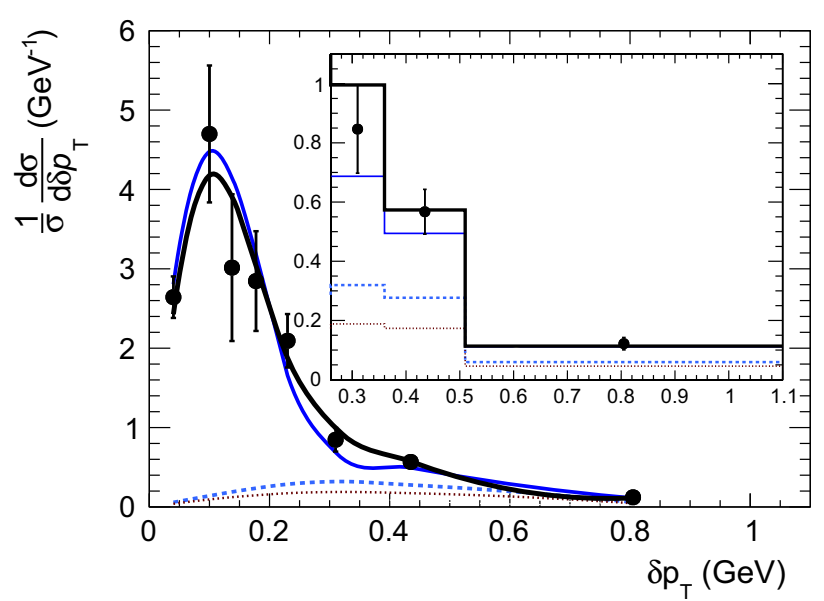

butions when using SuSAv2 and Valencia models is also displayed. A shape only comparison is also shown (right panel). Goodness of fit are calculated as follows. For $\delta p_{T}$ : $\chi_{\text {SuSA }}^{2}=20.5, \chi_{\text {Valencia }}^{2}=27.1$. The data points are taken from [98]. Figure adapted from Ref. [54]

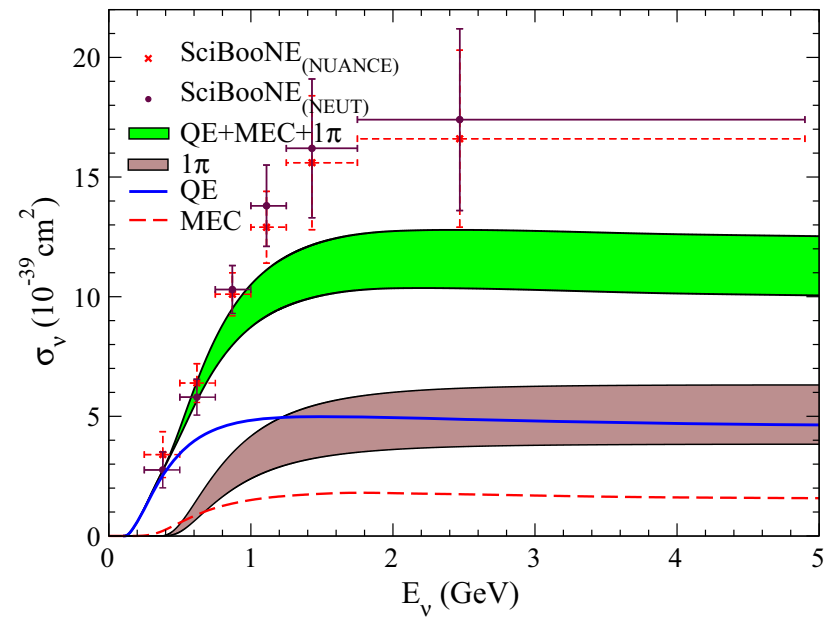

SciBooNE [103]. Theoretical predictions for QE, non-QE $(1 \pi)$ and the $2 \mathrm{p} 2 \mathrm{~h}$ MEC are shown separately. Plots from Ref. [18]

SuSA- $\Delta$ approach. Although an overall good agreement with data is observed in both panels, some underestimations are present at large kinematics, i.e., high $E_{\nu}$ and $Q_{Q E}^{2}$, which reveal the need for including higher resonances and deep inelastic scattering in the description. To address this, the SuSAv2-inelastic, which has been successfully applied for the analysis of the full inelastic spectrum in electron scattering (see Sect. 2.4 for details), will be soon extended to the analysis of the neutrino sector. 


\section{Conclusions}

In this paper, we have summarized the basic ingredients that go into scaling analyses of inclusive electron scattering and charge-changing neutrino reactions with nuclei. Importantly, good agreement in the former case is viewed as a pre-requisite to being able to predict the latter - clearly if one fails to account for inclusive electron scattering then it is unreasonable to expect that inclusive $\mathrm{CC} \nu$ reactions will be adequately modeled.

In particular, we have emphasized the use of scaling of the first kind (independence of $q$ at high energies) and of scaling of the second kind (independence of nuclear species). When both kinds of scaling are invoked we refer to it as super-scaling and accordingly our present focus has been placed on the so-called super-scaling approach (SuSA) in its original form and in extensions of that original form. Such extensions have been introduced to account for the modest level of scaling violations seen in direct comparisons with inclusive electron scattering data. In addition we have introduced the way $2 \mathrm{p}-2 \mathrm{~h}$ MEC effects have been incorporated, showing that, while they are usually corrections to the cross sections being represented via the scaling functions obtained either phenomenologically or through use of specific models, they typically are required to get a successful picture of the response.

We have briefly summarized these basic ideas and then proceeded to show examples of the excellent agreement found with inclusive electron scattering cross sections measured for several light to medium-weight nuclei. To place the discussions in context, we have also provided a section outlining how semi-inclusive scattering and inclusive scattering are related, and specifically how the former probes particular regions of missingenergy and -momentum (characterized by so-called trajectories), while the latter involves integrations over the $E_{m}-p_{m}$ plane of the semi-inclusive response. On the one hand, inclusive scattering, being a total hadronic cross section, is less dependent on the underlying details of nuclear structure. In fact, given that the kinematics of the single-nucleon knockout are handled relativistically and that there are sum rules that determine the integral of the inclusive response, then a single parameter can be used to fix the width of the response. For this reason many models yield the rough behaviour of the inclusive cross section, even the relativistic Fermi gas model. On the other hand, the semi-inclusive cross section depends critically on how the strength is distributed as a function of $E_{m}$ and $p_{m}$. Accordingly, models that fare reasonably well for inclusive scattering cannot be relied upon to properly represent the underlying nuclear structure needed for semi-inclusive reactions.

Finally, having discussed the foundations of superscaling for electron scattering we show some selected results for $\mathrm{CC} \nu$ reactions. The agreements with existing data are found to be excellent, giving us confidence that at least for inclusive neutrino reactions the problem appears to be well in hand. Note, however, from the statements made in the previous paragraph, that this is not to be taken as proof that semi-inclusive $\mathrm{CC} \nu$ modeling should be expected to be robust. Indeed, different models yield quite different results for cross sections in which both a charged lepton and a nucleon are detected in coincidence (a trend in modern experiments), even when the corresponding inclusive cross sections do not differ significantly.

The modeling of semi-inclusive reactions together with the analysis of nuclear-medium effects for different targets are part of our present projects. These have become essential ingredients for current neutrino experiments, being the origin of major experimental uncertainties on neutrino energy reconstruction and oscillation analyses. These aspects will be also of paramount relevance for the next generation of oscillation experiments, HyperKamiokande [104] and DUNE [105], which will enlarge the capabilities of current neutrino facilities to produce high-precision measurements and gain enough sensitivity to improve the determination of neutrino oscillation parameters, mass hierarchy and $\mathrm{CP}$ violating phase and where energy reconstruction and nuclear-medium uncertainties could be an important limiting factor.

Acknowledgements This work was supported in part by the Project BARM-RILO-20 of University of Turin and from INFN, National Project NUCSYS (M.B.B.); by the Madrid Government and Complutense University under, project PR65/19-22430 (R.G.-J.); by the Spanish Ministerio de Ciencia, Innovación y Universidades and ERDF (European Regional Development Fund) under contracts FIS201788410-P, and by the Junta de Andalucia (grants No. FQM160 and SOMM17/6105/UGR) (J.A.C.); by the University of Tokyo ICRR's Inter-University Research Program FY2020\&FY2021 and by the European Union's Horizon 2020 research and innovation programme under the Marie Skłodowska-Curie grant agreement No. 839481 (G.D.M.); by the Spanish Ministry of Science through grant FIS201785053-C2-1-P, and by Junta de Andalucia (grant No. FQM225) (J.E.A. and I.R.-S); by the Office of Nuclear Physics of the US Department of Energy under Grant Contract DEFG02-94ER40818 (T.W.D.).

Funding Open access funding provided by Università degli Studi di Torino within the CRUI-CARE Agreement.

Open Access This article is licensed under a Creative Commons Attribution 4.0 International License, which permits use, sharing, adaptation, distribution and reproduction in any medium or format, as long as you give appropriate credit to the original author(s) and the source, provide a link to the Creative Commons licence, and indicate if changes were made. The images or other third party material in this article are included in the article's Creative Commons licence, unless indicated otherwise in a credit line to the material. If material is not included in the article's Creative Commons licence and your intended use is not permitted by statutory regulation or exceeds the permitted use, you will need to obtain permission directly from the copyright holder. To view a copy of this licence, visit http://creativecomm ons.org/licenses/by/4.0/. 


\section{References}

1. K. Abe et al., T2K coll., Nature 580, 339 (2020) (Erratum in Nature 583, E16 (2020))

2. L. Alvarez-Ruso et al., Prog. Part. Nucl. Phys. 100, 1-68 (2018)

3. H. Gallagher, G. Garvey, G.P. Zeller, Annu. Rev. Nucl. Part. Sci. 61, 355-378 (2011)

4. J.G. Morfin, J. Nieves, J.T. Sobczyk, Adv. High Energy Phys. 2012, 934597 (2012)

5. J.A. Formaggio, G.P. Zeller, Rev. Mod. Phys. 84, 1307-1341 (2012)

6. L. Alvarez-Ruso, Y. Hayato, J. Nieves, New J. Phys. 16, 075015 (2014)

7. U. Mosel, Annu. Rev. Nucl. Part. Sci. 66, 171 (2016)

8. A.M. Ankowski, C. Mariani, J. Phys. G44, 054001 (2017)

9. O. Benhar, P. Huber, C. Mariani, D. Meloni, Phys. Rep. 700, 1 (2017)

10. T. Katori, M. Martini, J. Phys. G 45(1), 013001 (2018)

11. J. Bjorken, Phys. Rev. 179, 1547 (1969)

12. D.B. Day, J.S. McCarthy, T.W. Donnelly, I. Sick, Annu. Rev. Nucl. Part. Sci. 40, 357 (1990)

13. T.W. Donnelly, I. Sick, Phys. Rev. Lett. 82, 3212 (1999)

14. T.W. Donnelly, I. Sick, Phys. Rev. C 60, 065502 (1999)

15. J.E. Amaro, M.B. Barbaro, J.A. Caballero, T.W. Donnelly, A. Molinari, I. Sick, Phys. Rev. C 71, 015501 (2005)

16. R. González-Jiménez, G.D. Megias, M.B. Barbaro, J.A. Caballero, T.W. Donnelly, Phys. Rev. C 90, 035501 (2014)

17. G.D. Megias, J.E. Amaro, M.B. Barbaro, J.A. Caballero, T.W. Donnelly, I. Ruiz Simo, Phys. Rev. D 94, 093004 (2016)

18. J.E. Amaro, M.B. Barbaro, J.A. Caballero, R. González-Jiménez, G.D. Megias, I.R. Simo, J. Phys. G Nucl. Part. Phys. 47, 124001 (2020)

19. M.B. Barbaro, A. De Pace, L. Fiume, Universe 7(5), $140(2021)$

20. J.J. Kelly, Adv. Nucl. Phys. 23, 75 (1996)

21. J.E. Amaro, M.B. Barbaro, J.A. Caballero, T.W. Donnelly, J.M. Udías, Phys. Rev. C 75, 034613 (2007)

22. C. Maieron, T.W. Donnelly, I. Sick, Phys. Rev. C 65, $025502(2002)$

23. M.B. Barbaro, R. Cenni, A. De Pace, T.W. Donnelly, A. Molinari, Nucl. Phys. A 643, 137 (1998)

24. J.A. Caballero, J.E. Amaro, M.B. Barbaro, T.W. Donnelly, C. Maieron, J.M. Udías, Phys. Rev. Lett. 95, $252502(2005)$

25. J.A. Caballero, Phys. Rev. C 74, 054603 (2006)

26. J. Jourdan, Nucl. Phys. A 603, 117 (1996)

27. J.E. Amaro, M.B. Barbaro, J.A. Caballero, T.W. Donnelly, Phys. Rev. Lett. 98, 242501242501 (2007)

28. J.A. Caballero, J.E. Amaro, M.B. Barbaro, T.W. Donnelly, J.M. Udías, Phys. Lett. B 653, 366 (2007)

29. G.D. Megias, J.E. Amaro, M.B. Barbaro, J.A. Caballero, T.W. Donnelly, Phys. Rev. D 94, 013012 (2016)

30. R. González-Jiménez, A. Nikolakopoulos, N. Jachowicz, J.M. Udías, Phys. Rev. C 100, 045501 (2019)

31. R. González-Jiménez, M.B. Barbaro, J.A. Caballero, T.W. Donnelly, N. Jachowicz, G.D. Megias, K.
Niewczas, A. Nikolakopoulos, J.M. Udías, Phys. Rev. C 101, 015503015503 (2020)

32. M.B. Barbaro, J.A. Caballero, T.W. Donnelly, C. Maieron, Phys. Rev. C 69, 035502035502 (2004)

33. P.E. Bosted, M.E. Christy, Phys. Rev. C 77, 065206 (2008)

34. M.E. Christy, P.E. Bosted, Phys. Rev. C 81, 055213 (2010)

35. A. Bodek, J.L. Ritchie, Phys. Rev. D 23, 1070 (1981)

36. A. Bodek, J.L. Ritchie, Phys. Rev. D 24, 1400 (1981)

37. M. Glueck, E. Reya, A. Vogt, Eur. Phys. J 5, 461 (1998)

38. A. Bodek, U. Yang, arXiv:hep-ph/1011.6592 (2013)

39. O. Benhar, D. Day, I. Sick, Rev. Mod. Phys. 80, 189 (2008)

40. O. Benhar, D. Day, I. Sick, arXiv:nucl-ex/0603032 (2006)

41. G.D. Megias, Charged-current neutrino interactions with nucleons and nuclei at intermediate energies, Ph.D. Thesis, University of Seville, Spain (2017)

42. G.D. Megias, M.B. Barbaro, J.A. Caballero, J.E. Amaro, T.W. Donnelly, I. Ruiz Simo, J.W. Van Orden, J. Phys. G 46, 015104 (2019)

43. G.D. Megias, M.B. Barbaro, J.A. Caballero, S. Dolan, Phys. Rev. D 99, 113002 (2019)

44. C. Maieron, J.E. Amaro, M.B. Barbaro, J.A. Caballero, T.W. Donnelly, C.F. Williamson, Phys. Rev. C 80, 035504 (2009)

45. M.V. Ivanov, G.D. Megias, R. González-Jiménez, O. Moreno, M.B. Barbaro, J.A. Caballero, T.W. Donnelly, J. Phys. G Nucl. Part. Phys. 43, 045101 (2016)

46. M. Martini, M. Ericson, G. Chanfray, J. Marteau, Phys. Rev. C 80, 065501 (2009)

47. J. Nieves, I. Ruiz Simo, M.J. Vicente Vacas, Phys. Rev. C 83, 045501045501 (2011)

48. N. Rocco, C. Barbieri, O. Benhar, A. De Pace, A. Lovato, Phys. Rev. C 99, 025502 (2019)

49. J.E. Sobczyk, J. Nieves, F. Sanchez, Phys. Rev. C 102, $024601(2020)$

50. I. Ruiz Simo, J.E. Amaro, M.B. Barbaro, A. De Pace, J.A. Caballero, T.W. Donnelly, J. Phys. G 44, 065105 (2017)

51. E. Hernandez, J. Nieves, M. Valverde, Phys. Rev. D 76, 03305 (2007)

52. I. Ruiz Simo, C. Albertus, J.E. Amaro, M.B. Barbaro, J.A. Caballero, T.W. Donnelly, Phys. Rev. D 90, $033012(2014)$

53. I. Ruiz Simo, C. Albertus, J.E. Amaro, M.B. Barbaro, J.A. Caballero, T.W. Donnelly, Phys. Rev. D 90, 053010 (2014)

54. S. Dolan, G.D. Megias, S. Bolognesi, Phys. Rev. D 101, 033003 (2020)

55. I. Ruiz Simo, J.E. Amaro, M.B. Barbaro, J.A. Caballero, G.D. Megias, T.W. Donnelly, Phys. Lett. B 770, 193 (2017)

56. I. Ruiz Simo, J.E. Amaro, M.B. Barbaro, J.A. Caballero, G.D. Megias, T.W. Donnelly, Ann. Phys. 388, 323 (2018)

57. M.B. Barbaro, J.A. Caballero, A. De Pace, T.W. Donnelly, R. González-Jiménez, G.D. Megias, Phys. Rev. C 99, 042501 (2019)

58. M. Anghinolfi et al., Nucl. Phys. A 602, 405-422 (1996)

59. H. Dai et al., Phys. Rev. C 99, 054608 (2019) 
60. J.W. Van Orden, T.W. Donnelly, Phys. Rev. C 100, 044620 (2019)

61. R. González-Jiménez, M.B. Barbaro, J.A. Caballero, T.W. Donnelly, N. Jachowicz, G.D. Megias, K. Niewczas, A. Nikolakopoulos, J.W. Van Orden, J.M. Udías, arXiv:2104.01701 [nucl-th] (2021)

62. O. Benhar, A. Fabrocini, S. Fantoni, I. Sick, Nucl. Phys. A 579, 493 (1994)

63. O. Benhar, N. Farina, H. Nakamura, M. Sakuda, R. Seki, Phys. Rev. D 72, 053005 (2005)

64. K. Amir-Azimi-Nili, J. Udias, H. Müther, L. Skouras, A. Polls, Nucl. Phys. A 625, 633 (1997)

65. M.V. Ivanov, A.N. Antonov, G.D. Megias, J.A. Caballero, M.B. Barbaro, J.E. Amaro, I. Ruiz Simo, T.W. Donnelly, J.M. Udías, Phys. Rev. C 99, 014610 (2019)

66. J.M. Franco-Patino, J. Gonzalez-Rosa, J.A. Caballero, M.B. Barbaro, Phys. Rev. C 102, 064626 (2020)

67. O. Moreno, T.W. Donnelly, J.W. Van Orden, W.P. Ford, Phys. Rev. D 90, 013014 (2014)

68. K. Abe et al., Phys. Rev. D 93, 112012 (2016)

69. K. Abe et al., Phys. Rev. D 97, 012001 (2018)

70. J.E. Amaro, M.B. Barbaro, J.A. Caballero, A. De Pace, T.W. Donnelly, G.D. Megias, I. Ruiz Simo, Phys. Rev. C 95, 065502 (2017)

71. K. Abe et al., Phys. Rev. D 101, 112004 (2020)

72. C.E. Patrick et al., Phys. Rev. D 97, 052002 (2018)

73. D. Ruterbories et al., Phys. Rev. D 99, 012004 (2019)

74. C. Andreopoulos et al., Nucl. Instrum. Methods A 614, $87(2010)$

75. G.D. Megias, S. Bolognesi, M.B. Barbaro, E. TomasiGustafsson, Phys. Rev. C 101, 025501 (2020)

76. J. Nieves, I. Ruiz Simo, M.J. Vicente Vacas, Phys. Lett. B 707, 72 (2012)

77. M. Martini, M. Ericson, G. Chanfray, J. Marteau, Phys. Rev. C 81, 045502 (2010)

78. M. Martini, M. Ericson, G. Chanfray, Phys. Rev. D 85, 093012 (2012)

79. K. Gallmeister, U. Mosel, J. Weil, Phys. Rev. C 94, 035502 (2016)

80. A. Meucci, C. Giusti, Phys. Rev. D 85, 093002 (2012)

81. A. Meucci, C. Giusti, Phys. Rev. D 91, 093004 (2015)
82. N. Rocco, A. Lovato, O. Benhar, Phys. Rev. Lett. 116, $192501(2016)$

83. T. Golan, C. Juszczak, J.T. Sobczyk, Phys. Rev. C 86, 015505015505 (2012)

84. A. Lovato, S. Gandolfi, J. Carlson, S.C. Pieper, R. Schiavilla, Phys. Rev. C 91, 062501 (2015)

85. V. Pandey, N. Jachowicz, M. Martini, R. GonzálezJiménez, J. Ryckebusch, T. Van Cuyck, N. Van Dessel, Phys. Rev. C 94, 054609 (2016)

86. A.V. Butkevich, S.V. Luchuk, Phys. Rev. C 97, 045502 (2018)

87. M.V. Ivanov, A.N. Antonov, J.A. Caballero, G.D. Megias, M.B. Barbaro, E. Moya de Guerra, J.M. Udías, Phys. Rev. C 89, 014607014607 (2014)

88. A.V. Butkevich, S.V. Luchuk, Phys. Rev. D 99, 093001 (2019)

89. M. Martini, M. Ericson, G. Chanfray, Phys. Rev. C 84, $055502(2011)$

90. J. Nieves, I. Ruiz Simo, M.J. Vicente Vacas, Phys. Lett. B 721, 90 (2013)

91. M. Martini, M. Ericson, Phys. Rev. C 87, 065501 $065501(2013)$

92. U. Mosel, O. Lalakulich, K. Gallmeister, Phys. Rev. D 89, 093003 (2014)

93. A. Meucci, C. Giusti, Phys. Rev. D 89, 117301117301 (2014)

94. M. Ericson, M. Martini, Phys. Rev. C 91, 035501 (2015)

95. U. Mosel, K. Gallmeister, Phys. Rev. C 97, 045501 (2018)

96. S. Dolan, U. Mosel, K. Gallmeister, L. Pickering, S. Bolognesi, Phys. Rev. C 98, 045502 (2018)

97. Y. Hayato, Acta Phys. Pol. B 40, 2477 (2009)

98. K. Abe et al., Phys. Rev. D 98, 032003 (2018)

99. J.M. Franco-Patino, R. Gonzalez-Jimenez et al., (2021) (In preparation)

100. X.-G. Lu, L. Pickering, S. Dolan, G. Barr, D. Coplowe, Y. Uchida, D. Wark, M.O. Wascko, A. Weber, T. Yuan, Phys. Rev. C 94, 015503 (2016)

101. S. Dolan, arXiv:1810.06043 [hep-ex] (2018)

102. K. Abe et al., Phys. Rev. Lett. 113, 241803 (2014)

103. Y. Nakajima et al., Phys. Rev. D 83, 012005 (2011)

104. K. Abe et al., arXiv:1805.04163 [physics.ins-det] (2018)

105. B. Abi et al., arXiv:2002.02967 [physics.ins-det] (2020) 